\title{
Simulation of fluid flow and collection efficiency for an SEA multi-element probe
}

\author{
David L. Rigby \\ Vantage Partners Limited, Cleveland, OH, 44135 \\ Peter M. Struk \\ NASA Glenn Research Center, Cleveland, OH, 44135 \\ and \\ Colin Bidwell \\ NASA Glenn Research Center, Cleveland, OH, 44135
}




\section{Outline}

- Describe problem and simplifications

- Description of cases run

- Some views of the flowfield

- Heat transfer results

- Collection efficiency results 


\section{CAD Surface and Grid}
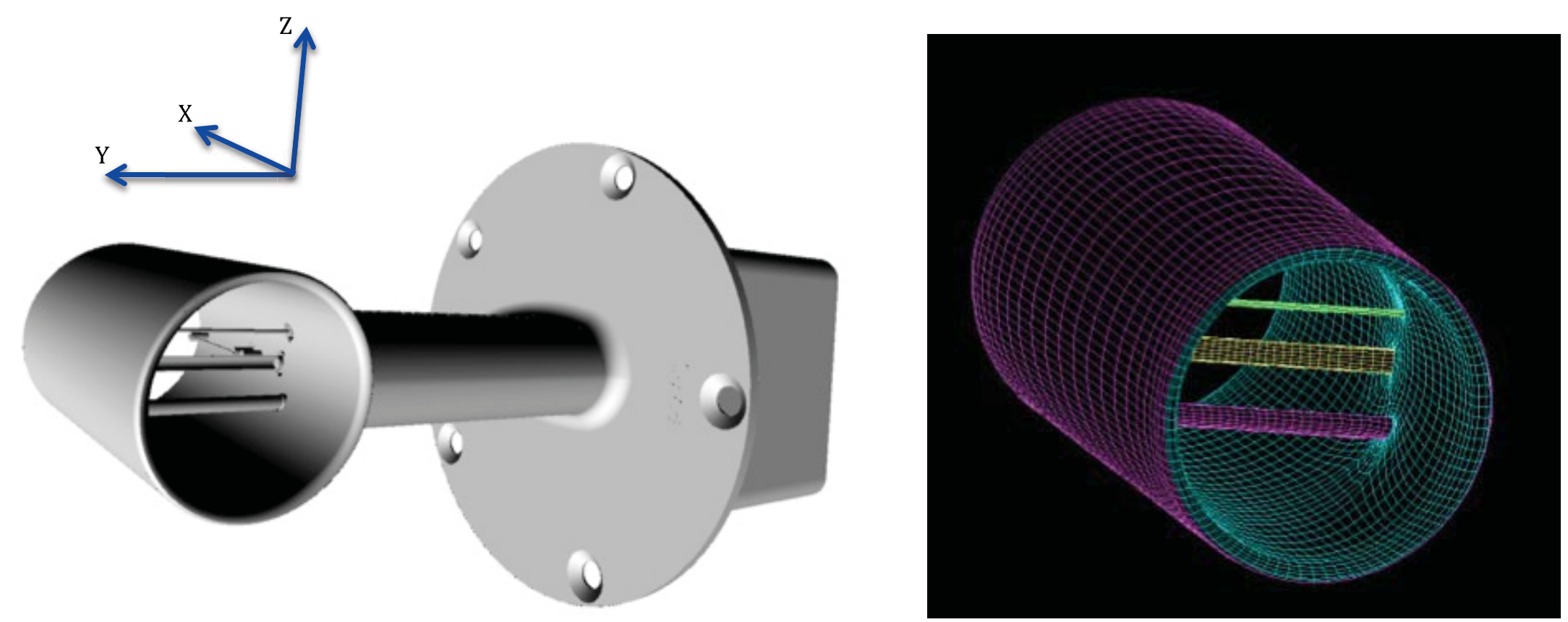

- CAD representation (left) and surface mesh for CFD simulation. Angle of attack refers to a rotation about the $Y$-axis. Yaw refers to a rotation about the $Z$-axis. 


\section{Schematic showing coordinate system}

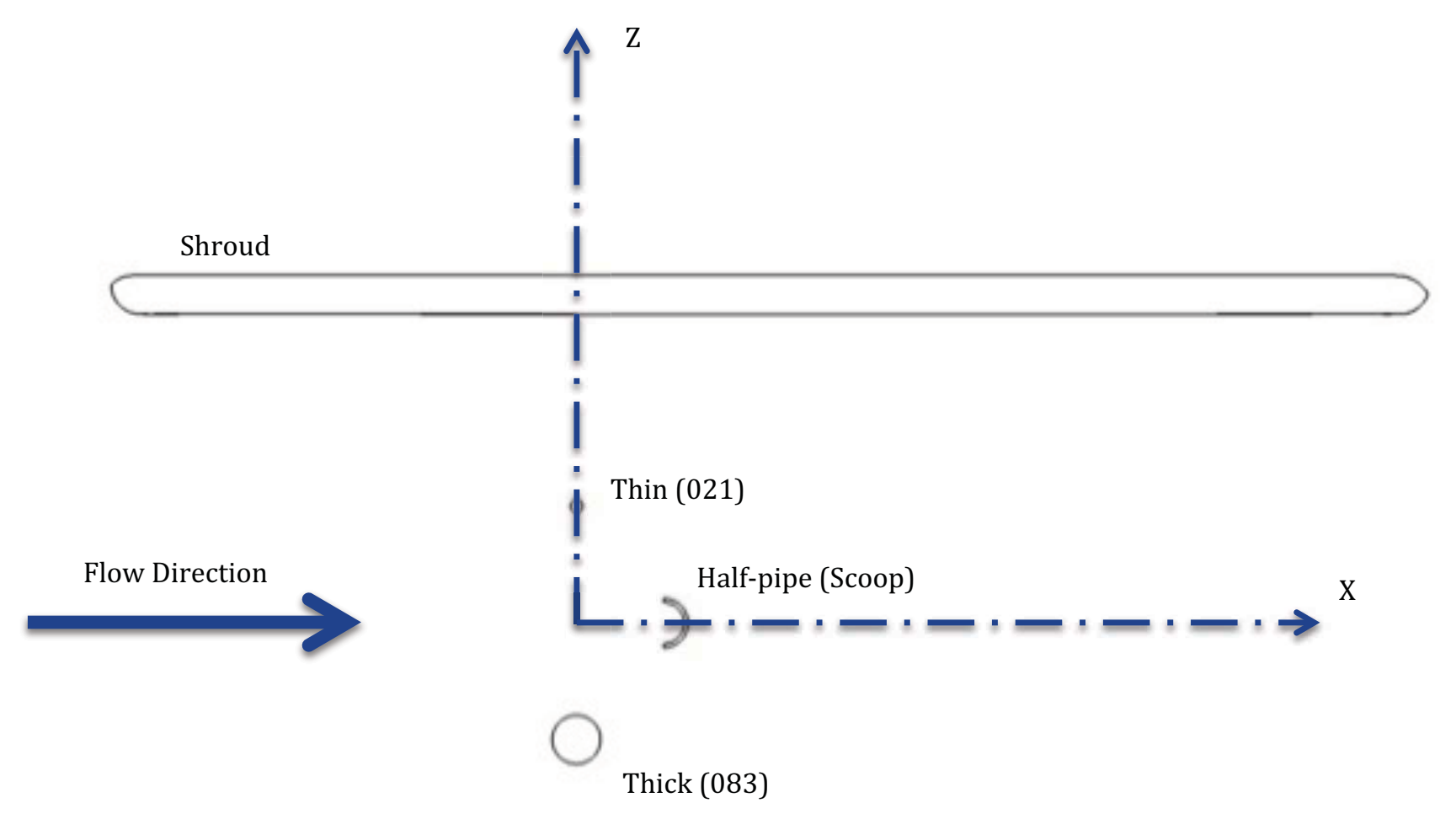

Shroud

- Thin wire has a diameter of 0.021 inches

- Thick wire has a diameter of 0.083 inches

- Half-pipe has same diameter as thick wire 


\section{Simplifications}

- Compensation wire is not modeled

- Mount is not included (in most cases)

- Wires and scoop enter shroud cleanly

- Domain much larger than wind tunnel (in most cases)

- 3D Unsteady Reynolds Averaged Navier-Stokes simulation

- Focus is on time averaging of the unsteady simulation

- Fixed wall temperature

- Each heated element is held at a fixed temperature of $140^{\circ} \mathrm{C}$

- The shroud is held at $50^{\circ} \mathrm{C}$

- Freestream static temperature is $-5^{\circ} \mathrm{C}$ for all cases 


\section{Description of Cases Run}

- Isolated, no support

- Straight on

- Total Pressure = 13.5 psi; Velocity $=85,100,135 \mathrm{~m} / \mathrm{s}$

- Total Pressure $=6.5$ psi; Velocity $=85,100,135 \mathrm{~m} / \mathrm{s}$

- Total Pressure $=13.5$ psi; Velocity $=85$

- Straight on, 5 degrees yaw, 5 degrees pitch

- In tunnel, with support

- Straight on

- Total Pressure $=13.5$ psi; Velocity $=85$ 


\section{Table of Cases}

\begin{tabular}{|l|r|r|r|r|c|c|}
\hline \multicolumn{1}{|c|}{ Name } & $P_{0}$ (psia) & $\begin{array}{c}\text { Velocity } \\
(\mathrm{m} / \mathrm{s})\end{array}$ & $\begin{array}{c}\alpha, \text { Pitch } \\
\text { (degrees) }\end{array}$ & $\begin{array}{c}\gamma, \text { Yaw } \\
\text { (degrees) }\end{array}$ & Support & Grid \\
\hline Pt06p5V085 & 6.5 & 85 & 0 & 0 & No & Normal \\
\hline Pt06p5V100 & 6.5 & 100 & 0 & 0 & No & Normal \\
\hline Pt06p5V135 & 6.5 & 135 & 0 & 0 & No & Normal \\
\hline Pt13p5V085 & 13.5 & 85 & 0 & 0 & No & Normal \\
\hline Pt13p5V100 & 13.5 & 100 & 0 & 0 & No & Normal \\
\hline Pt13p5V135 & 13.5 & 135 & 0 & 0 & No & Normal \\
\hline Attack5deg & 13.5 & 85 & 5 & 0 & No & Normal \\
\hline Yaw5deg & 13.5 & 85 & 0 & 5 & No & Normal \\
\hline Support & 13.5 & 85 & 0 & 0 & Yes & Normal \\
\hline Pt13p5V085c & 13.5 & 85 & 0 & 0 & No & Coarsened \\
\hline Pt13p5V085f & 13.5 & 85 & 0 & 0 & No & Refined \\
\hline
\end{tabular}




\section{Instantaneous vs Time-Averaged Result}

- Instantaneous Snapshot

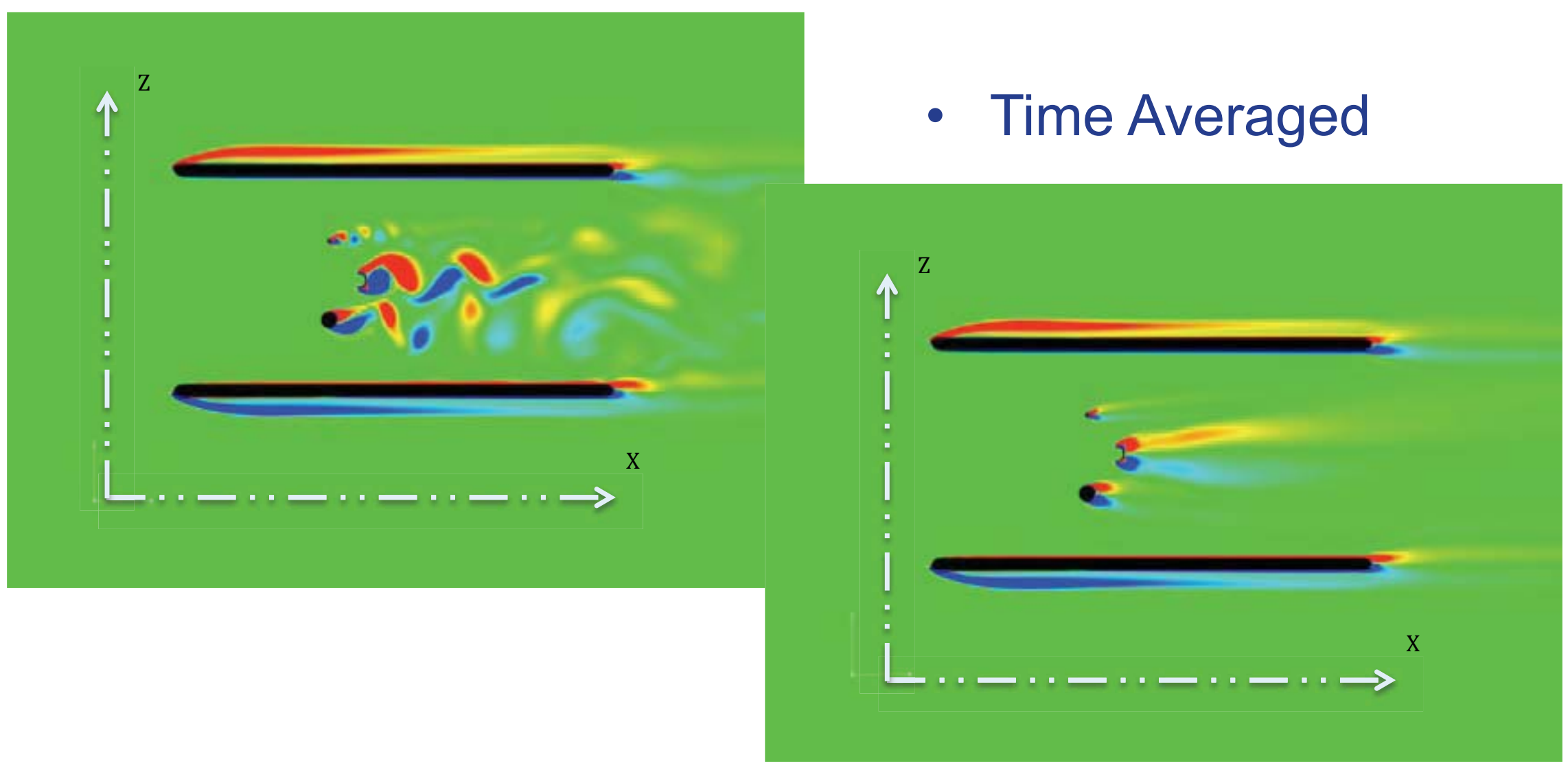

- Y-vorticity (red is positive, blue is negative) 


\section{Pressure and Axial Velocity}

- Pressure in symmetry plane and on surfaces

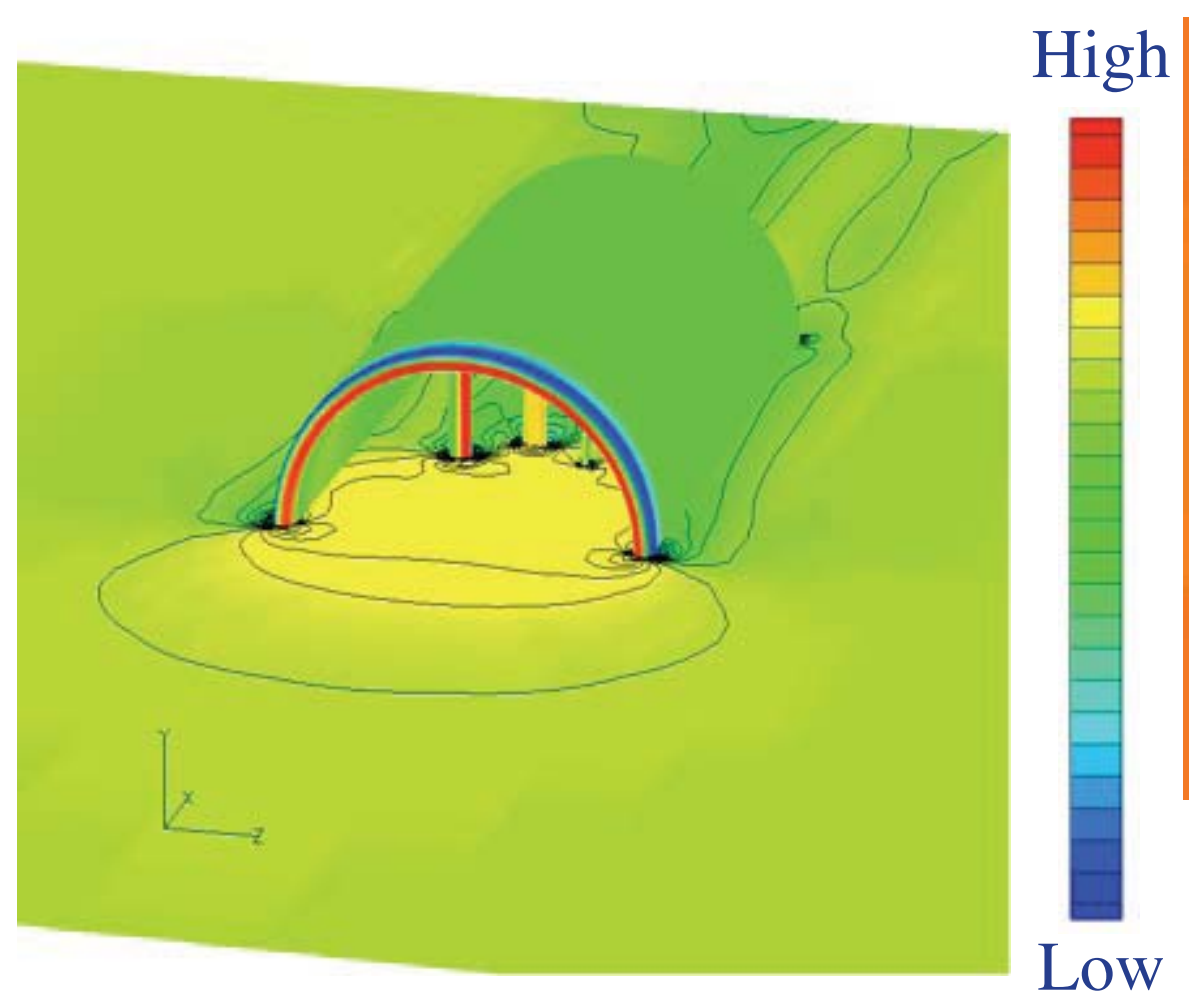

- Axial velocity in symmetry plane

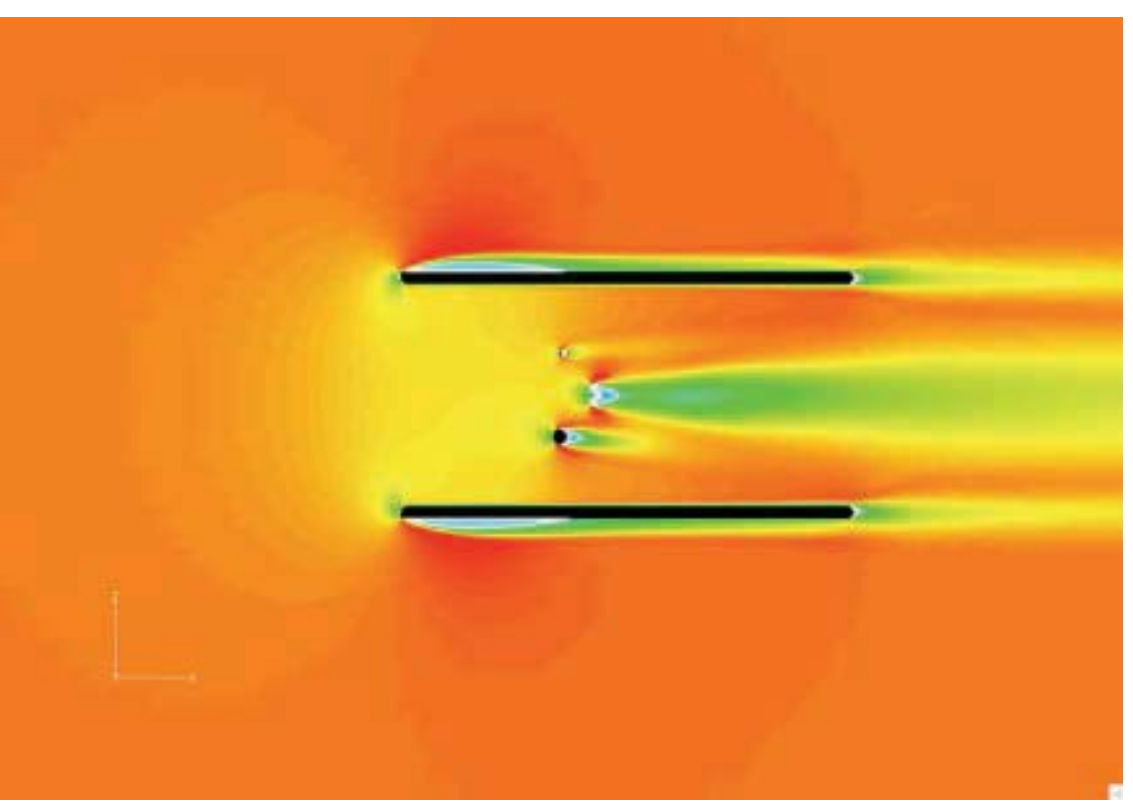

White lines, representing zero velocity, enclose reverse flow regions 


\section{Axial Velocity (closer view)}
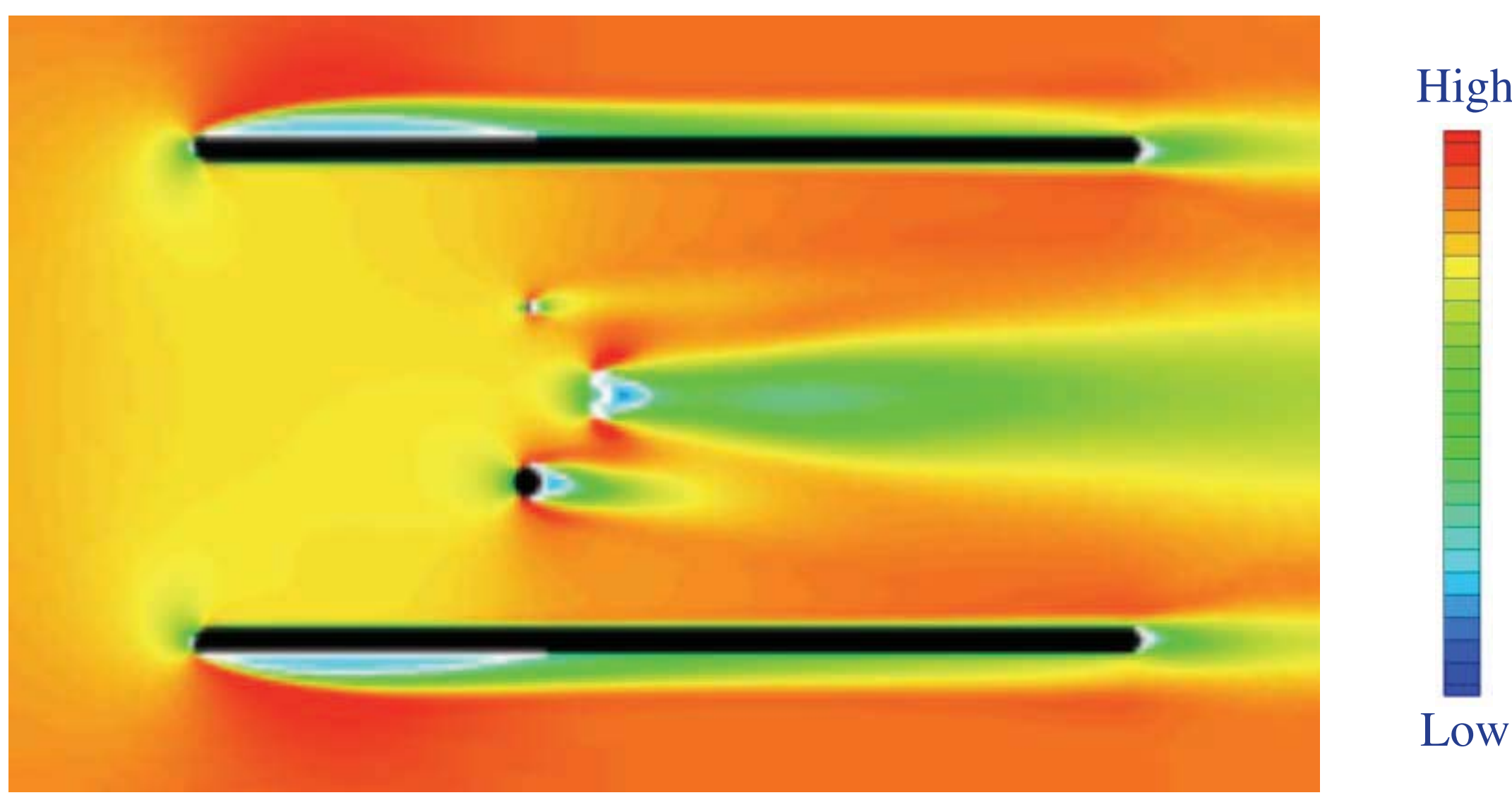

White lines, representing zero velocity, enclose reverse flow regions 


\section{Table of Shroud Mass Flux}

\begin{tabular}{|l|c|}
\hline \multicolumn{1}{|c|}{ Name } & $\begin{array}{c}\text { Normalized } \\
\text { Average Shroud } \\
\text { Mass Flux }\end{array}$ \\
\hline Pt06p5V085 & 0.7905 \\
\hline Pt06p5V100 & 0.7855 \\
\hline Pt06p5V135 & 0.7895 \\
\hline Pt13p5V085 & 0.7988 \\
\hline Pt13p5V100 & 0.7975 \\
\hline Pt13p5V135 & 0.7917 \\
\hline Attack5deg & 0.7977 \\
\hline Yaw5deg & 0.8045 \\
\hline Support & 0.8034 \\
\hline Pt13p5V085c & 0.8344 \\
\hline Pt13p5V085f & 0.7871 \\
\hline
\end{tabular}

- Shroud mass flux is approximately $80 \%$ of freestream mass flux for all cases 


\section{Heat Transfer Results}

- Presented in terms of Nusselt number

$-\mathrm{Nu}=h^{*} \mathrm{~d} / \mathrm{k}$

- h $\quad=q /\left(T_{\text {wall }}-T_{t, \infty}\right)$

- $\mathrm{q} \quad=$ wall heat flux

- $\mathrm{T}_{\text {wall }}=$ wall temperature

- $\mathrm{T}_{\mathrm{t}, \infty} \quad=$ freestream total temperature

- $\mathrm{d}=$ wire diameter

- $\mathrm{k}=$ air thermal conductivity

- Overall, as well as frontal results are generated

- Frontal results are of interest because particles only impact the front of each heated element

- Spanwise variation of the heat transfer is also investigated

- Angle of attack, yaw, and the case with the support produced little change in the heat transfer

- Details are in the paper, but not presented here in the interest of time 


\section{Nusselt number results for straight on cases}

- Overall refers to an average over the entire heated element

- Front refers to averaging over the upstream facing surface

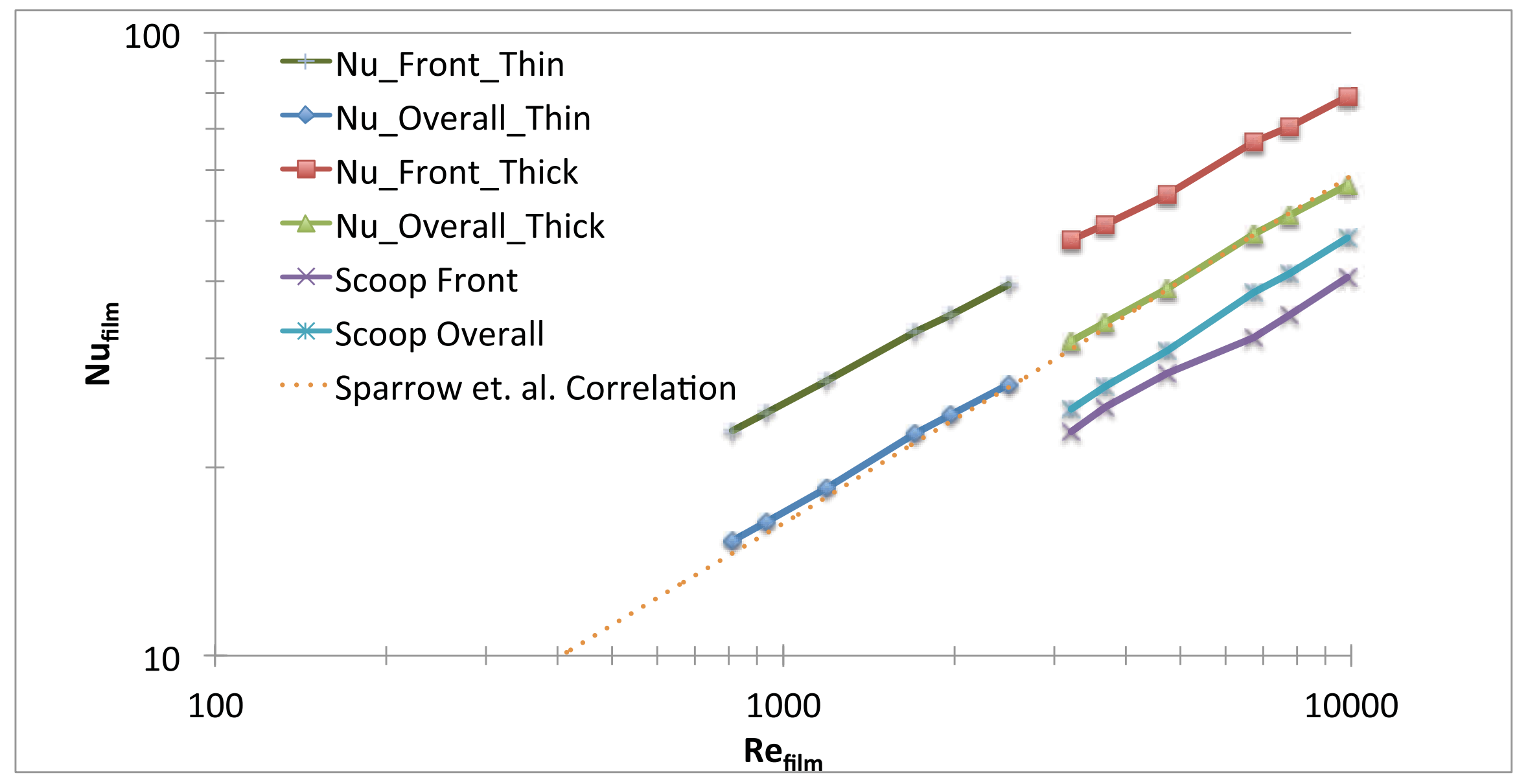




\section{Normalized Nusselt number averaged from all six straight on cases}

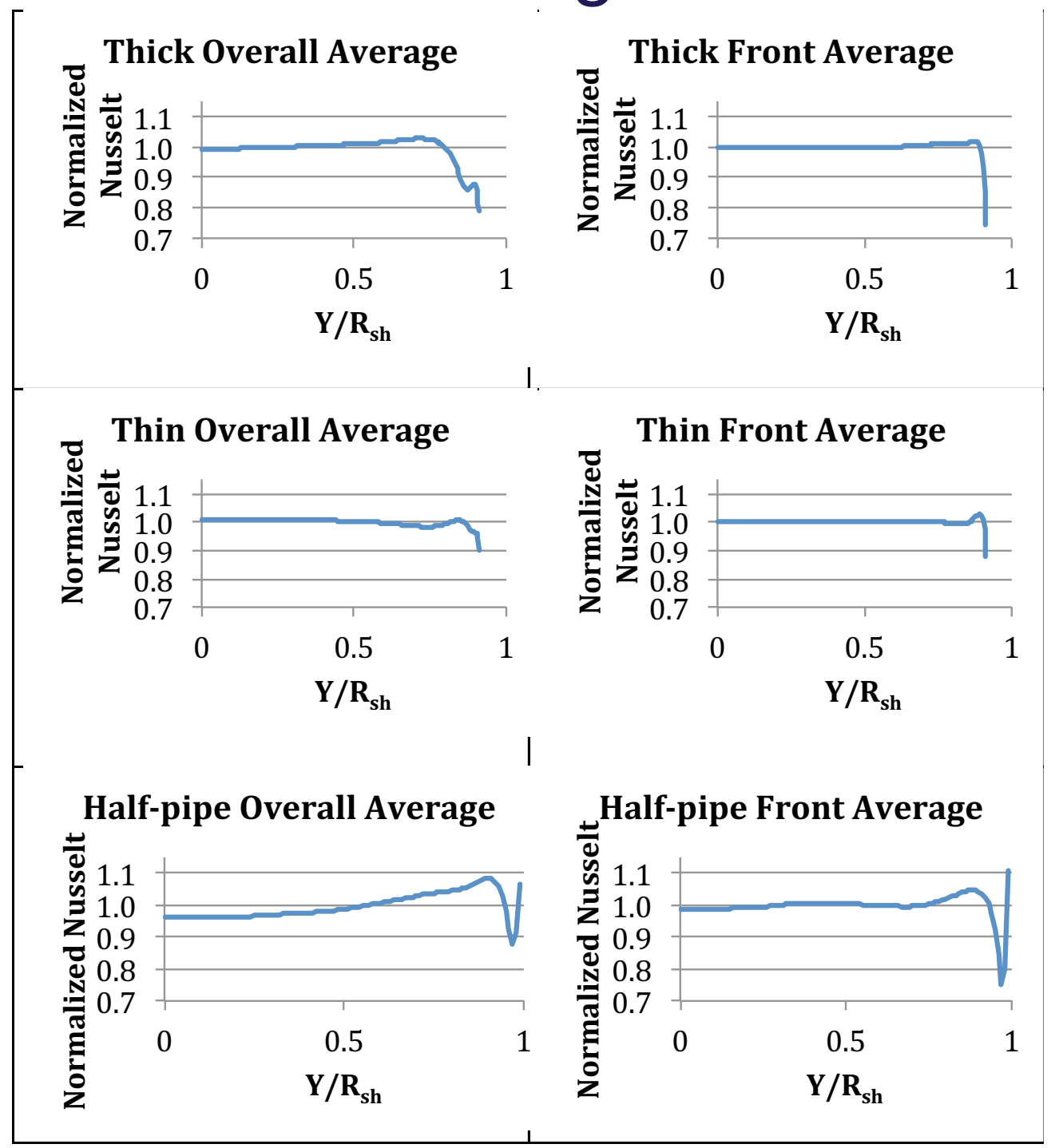




\section{Collection Efficiency Results}

- Collection efficiency is a calculated using the LEWICE3D code

- Uses the Navier-Stokes results from Glenn-HT

- Particles sizes of 5, 20, 50, and 100 microns are investigated

- Results from an SLD splashing model are also included for the 100 micron cases

- Both total and local collection efficiency results are presented

- Total collection efficiency, $E_{m}$, is the ratio of the particle mass impinging on a body to the free stream impingement rate through an area equal to the projected area of the body

- Local collection efficiency, $\beta$, is defined as the ratio of the surface mass flux of particles to the free stream mass flux of particles 


\section{Total versus Local Collection Efficiency (a visual explanation)}

Total Collection Efficiency, $\mathrm{E}_{\mathrm{m}}=\mathrm{L}_{\text {captured }} / \mathrm{L}_{\text {projected }}$

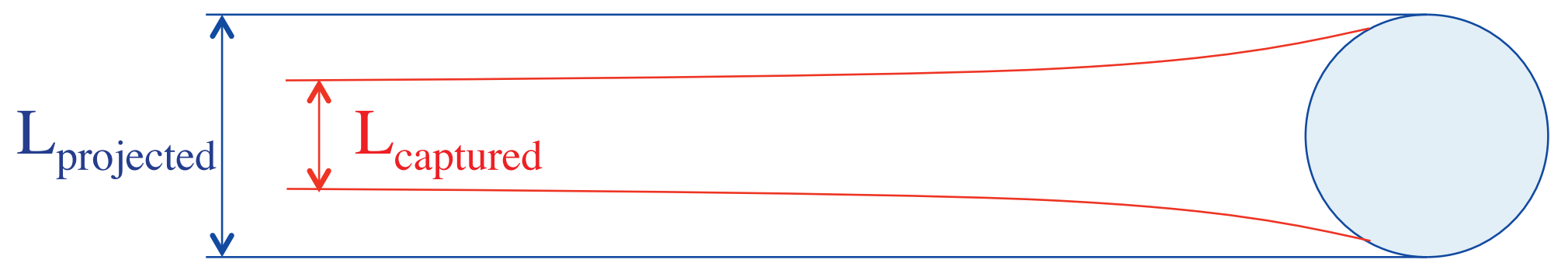

Local Collection Efficiency, $\beta=\mathrm{L}_{\infty} / \mathrm{S}$

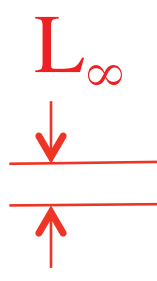




\section{Local Collection Efficiency (Looking from upstream)}
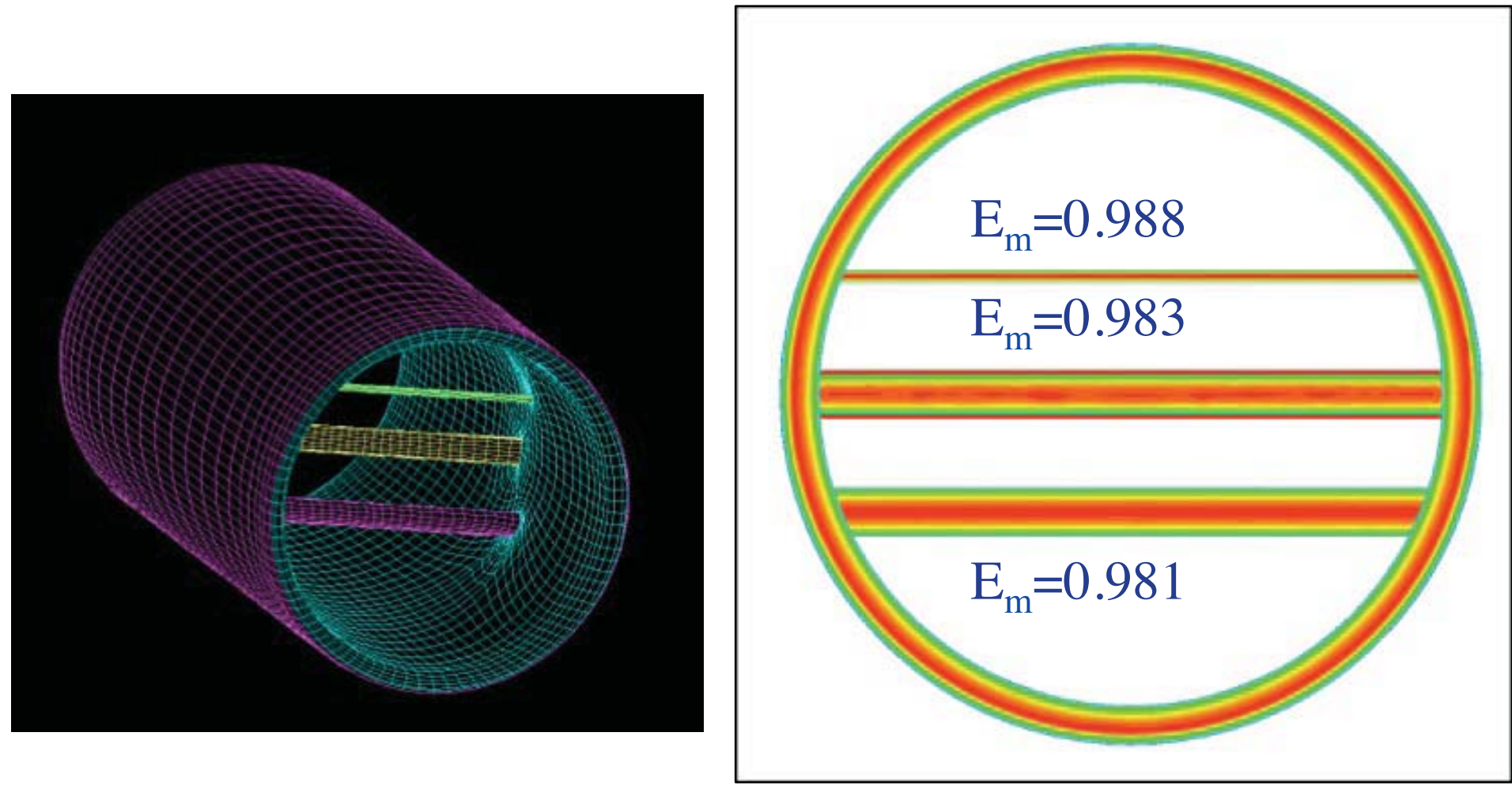

\begin{tabular}{|ll}
\hline & $\beta$ \\
1 & 1 \\
0.95 \\
0.9 \\
0.85 \\
0.8 \\
0.75 \\
0.7 \\
0.65 \\
0.6 \\
0.55 \\
0.5 \\
0.45 \\
0.4 \\
0.35 \\
0.3 \\
0.25 \\
0.2 \\
\hline 0.15 \\
0.1 \\
0.05 \\
0 \\
\hline
\end{tabular}




\section{Local Collection Efficiency for several particle sizes (straight on cases).}

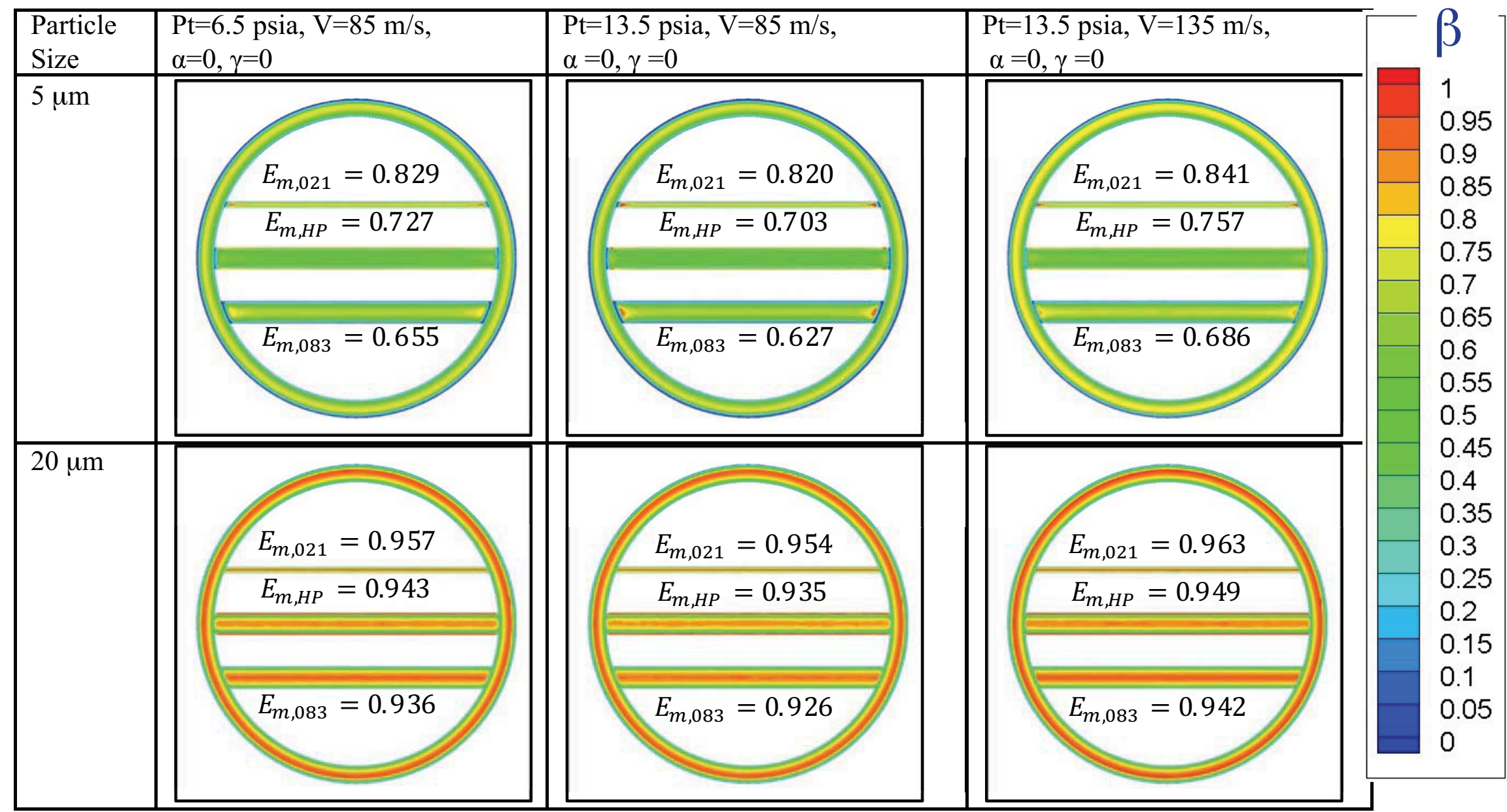




\section{Local Collection Efficiency for several particle sizes (straight on cases).}

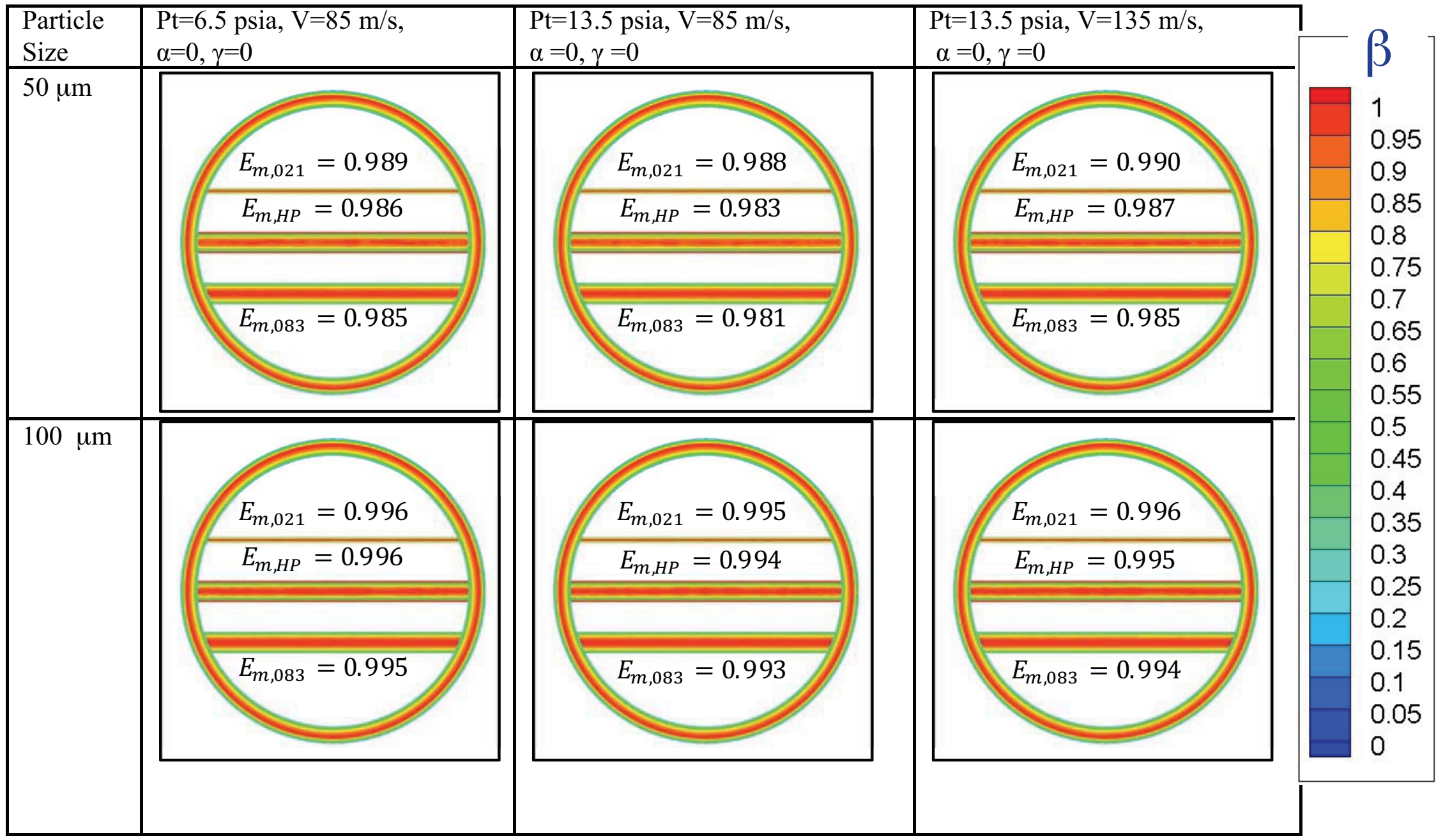


Local Collection Efficiency for several particle sizes (straight on cases).

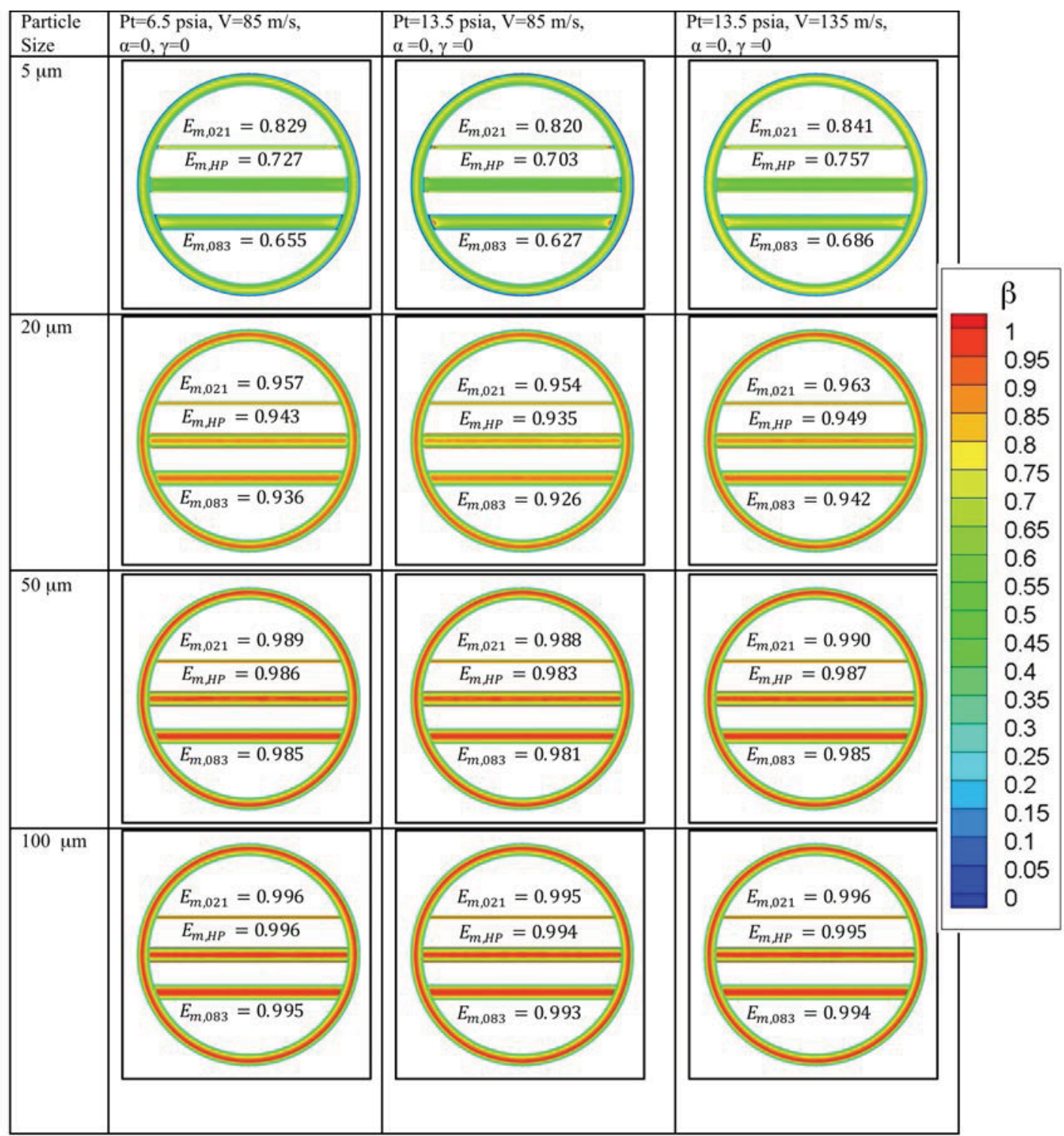


Collection efficiency for several particle sizes (angle of attack, yaw and support cases).

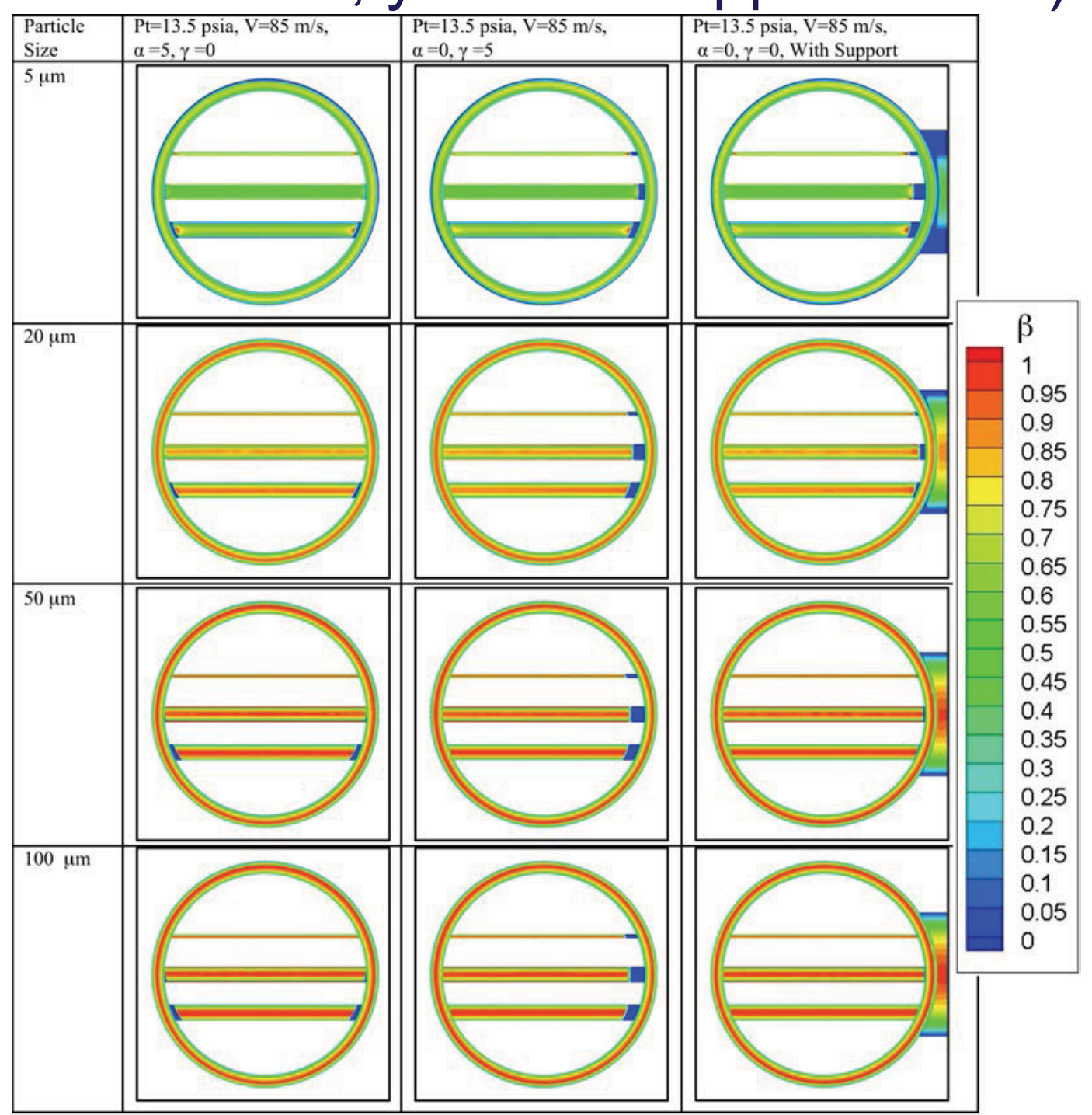




\section{Collection efficiency Splashing compared to no splashing}

$100 \mu \mathrm{m}$

$\mathrm{Pt}=13.5$ psia,

$\mathrm{V}=85 \mathrm{~m} / \mathrm{s}$, $\alpha=0, \gamma=0$
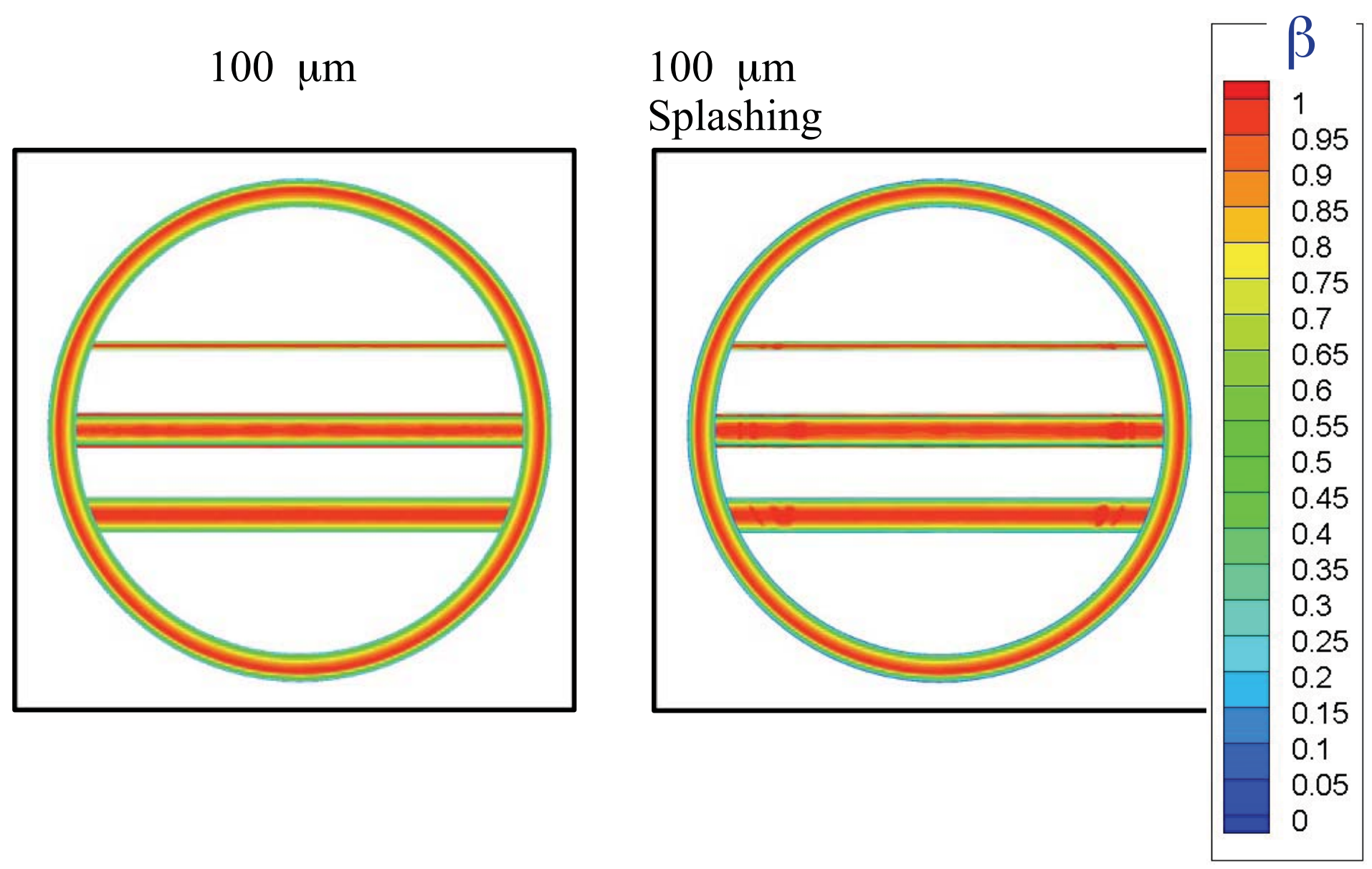
Total collection efficiency results for straight on cases without support Thick (083) Heated Element

\begin{tabular}{|l|c|c|c|r|r|r|r|}
\hline \multicolumn{1}{|c|}{ Geometry } & Total Pressure & \multicolumn{1}{c}{ Airspeed } & \multicolumn{1}{c}{ Drop Size } & \multicolumn{1}{c|}{ K_0 } & \multicolumn{1}{c|}{ E_m,2D } & \multicolumn{1}{c|}{ E_m,3D } & E_m,3D/E_m,2D \\
\hline & psia & $\mathrm{m} / \mathrm{s}$ & Microns & & Lewice2D & Lewice3D & \\
\hline Thick (083) & 6.5 & 85 & 5 & 2.15 & 0.782 & 0.655 & $83.7 \%$ \\
\hline Thick (083) & 6.5 & 85 & 20 & 23.39 & 0.974 & 0.936 & $96.1 \%$ \\
\hline Thick (083) & 6.5 & 85 & 50 & 103.26 & 0.992 & 0.985 & $99.2 \%$ \\
\hline Thick (083) & 6.5 & 85 & 100 & 304.29 & 0.996 & 0.995 & $99.9 \%$ \\
\hline Thick (083) & 13.5 & 85 & 5 & 1.79 & 0.754 & 0.627 & $83.1 \%$ \\
\hline Thick (083) & 13.5 & 85 & 20 & 17.82 & 0.968 & 0.926 & $95.7 \%$ \\
\hline Thick (083) & 13.5 & 85 & 50 & 74.75 & 0.990 & 0.981 & $99.1 \%$ \\
\hline Thick (083) & 13.5 & 85 & 100 & 213.24 & 0.995 & 0.993 & $99.8 \%$ \\
\hline Thick (083) & 13.5 & 135 & 5 & 2.53 & 0.816 & 0.686 & $84.0 \%$ \\
\hline Thick (083) & 13.5 & 135 & 20 & 24.02 & 0.975 & 0.942 & $96.6 \%$ \\
\hline Thick (083) & 13.5 & 135 & 50 & 98.33 & 0.992 & 0.985 & $99.2 \%$ \\
\hline Thick (083) & 13.5 & 135 & 100 & 276.34 & 0.995 & 0.994 & $99.9 \%$ \\
\hline
\end{tabular}




\section{Conclusions}

- Time average URANS results from Glenn-HT/ LEWICE3D provide reasonable prediction of heat transfer and collection efficiency for the SEA multielement probe

- Flow within shroud is approximately $80 \%$ of freestream flow for all cases

- Nusselt number results follow predictable trend lines as a function of Reynolds number

- Pitch, Yaw, and the inclusion of the support strut have little effect on average Nusselt number results

- Total collection efficiency of heated elements approaches $100 \%$ for 50 and 100 micron particles 


\section{Conclusions (continued)}

- Total collection efficiency is reduced as particle size decreases

- Decreased inertia parameter, as particle size decreases, explains much of reduction

- Additional reduction observed due to effect of shroud

- Additional reduction is quantified by comparing $3 \mathrm{D}$ and $2 \mathrm{D}$ results

- Reduced flow through shroud causes decrease in flux of particles (compared to freestream) approaching heated elements

- Reduced velocity within shroud allows particles to more easily avoid heated elements

- Effect of shroud becomes negligible as particle size increases 


\section{Thank You}




\section{Backup Slides}




\section{Grid Dependence of Shroud Mass Flux}

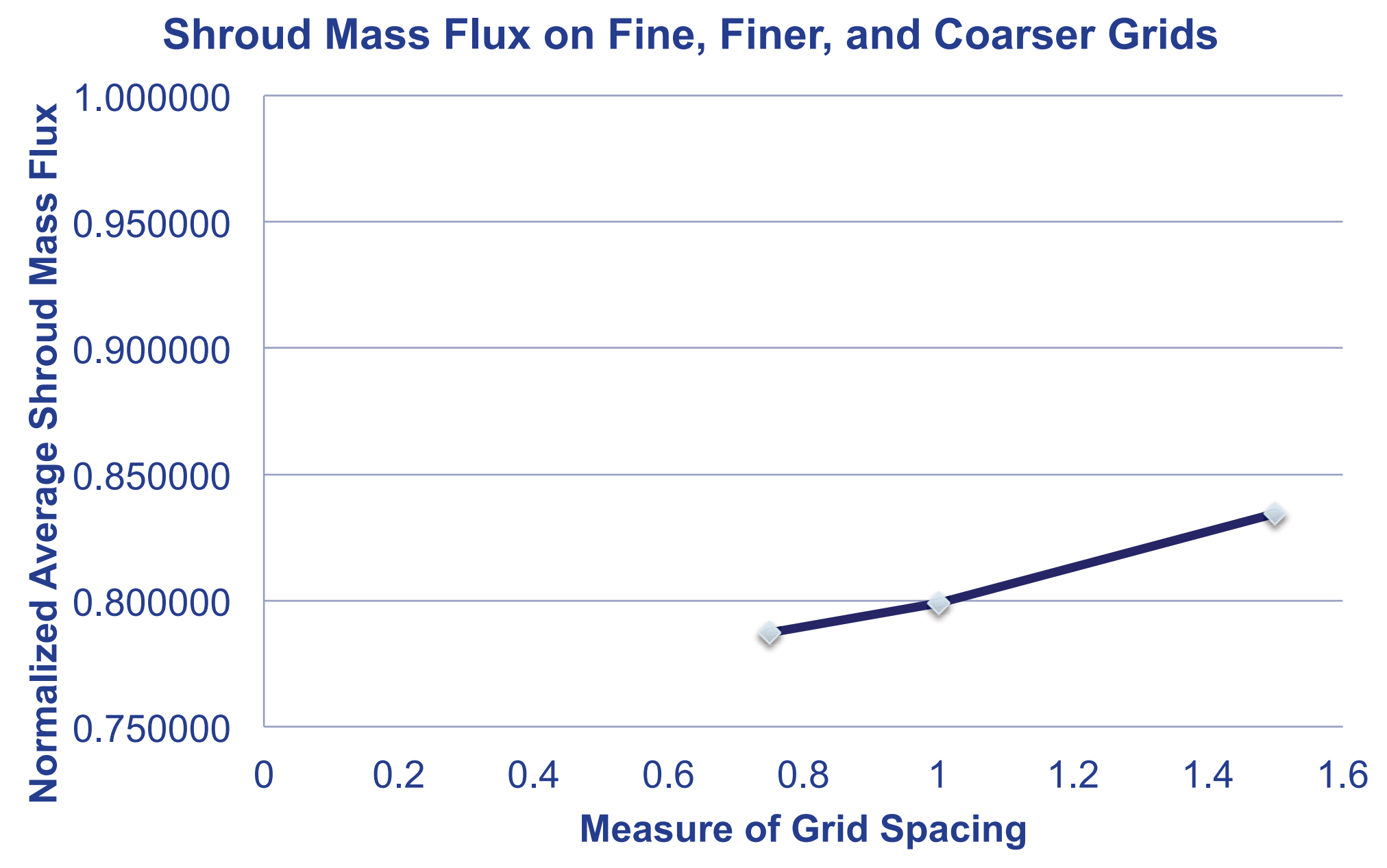




\section{Grid Dependence of Nusselt Numbers}

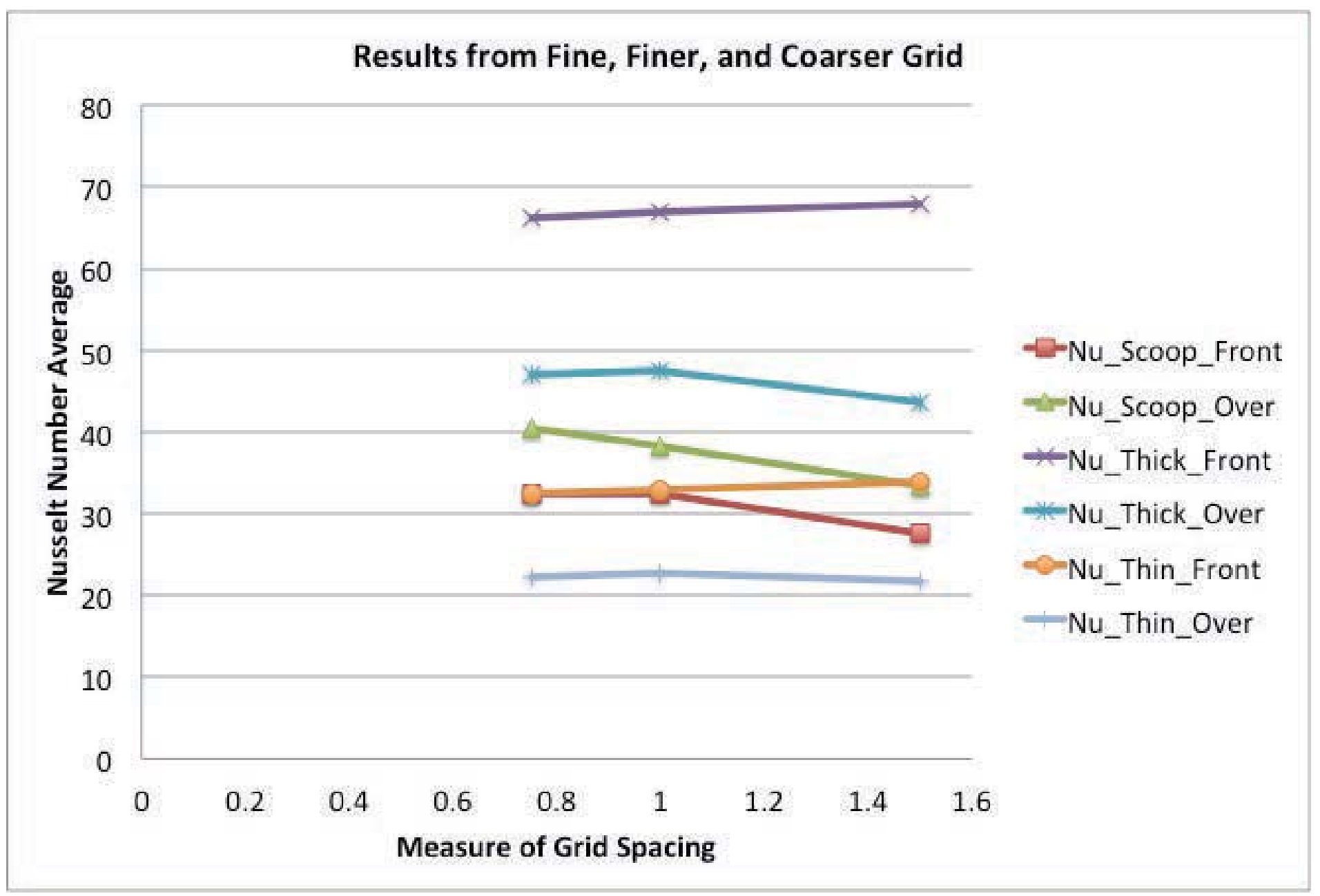




\section{Effect of Pitch, Yaw, and Support Strut on Average Nusselt numbers}

\begin{tabular}{|l|r|r|r|r|r|}
\hline Name & \multicolumn{1}{|l|}{$\mathrm{Re}_{\text {thick }}$} & Scoop Front & \multicolumn{1}{l|}{ Scoop Overall } & \multicolumn{1}{l|}{ Thick Front } & \multicolumn{1}{l|}{ Thick Overall } \\
\hline Pt13p5V085 & 6742 & 32.349 & 38.256 & 66.884 & 47.388 \\
\hline Attack5deg & 6733 & 32.154 & 38.132 & 66.957 & 47.333 \\
\hline Yaw5deg & 6790 & 32.110 & 37.691 & 67.178 & 47.528 \\
\hline Support & 6781 & 32.039 & 37.016 & 66.872 & 47.475 \\
\hline
\end{tabular}

Thick and Scoop average Nusselt numbers for other cases. (Thick and Scoop have the same diameter).

\begin{tabular}{|l|r|r|r|}
\hline Name & \multicolumn{1}{|l|}{ Re $_{\text {thin }}$} & \multicolumn{1}{l|}{ Thin Front } & \multicolumn{1}{l|}{ Thin Overall } \\
\hline Pt13p5V085 & 1706 & 32.992 & 22.662 \\
\hline Attack5deg & 1703 & 32.863 & 22.563 \\
\hline Yaw5deg & 1718 & 33.001 & 22.671 \\
\hline Support & 1716 & 32.970 & 22.643 \\
\hline
\end{tabular}

Thin average Nusselt number for other cases. 


\section{Thick (083) Spanwise Nusselt Number}
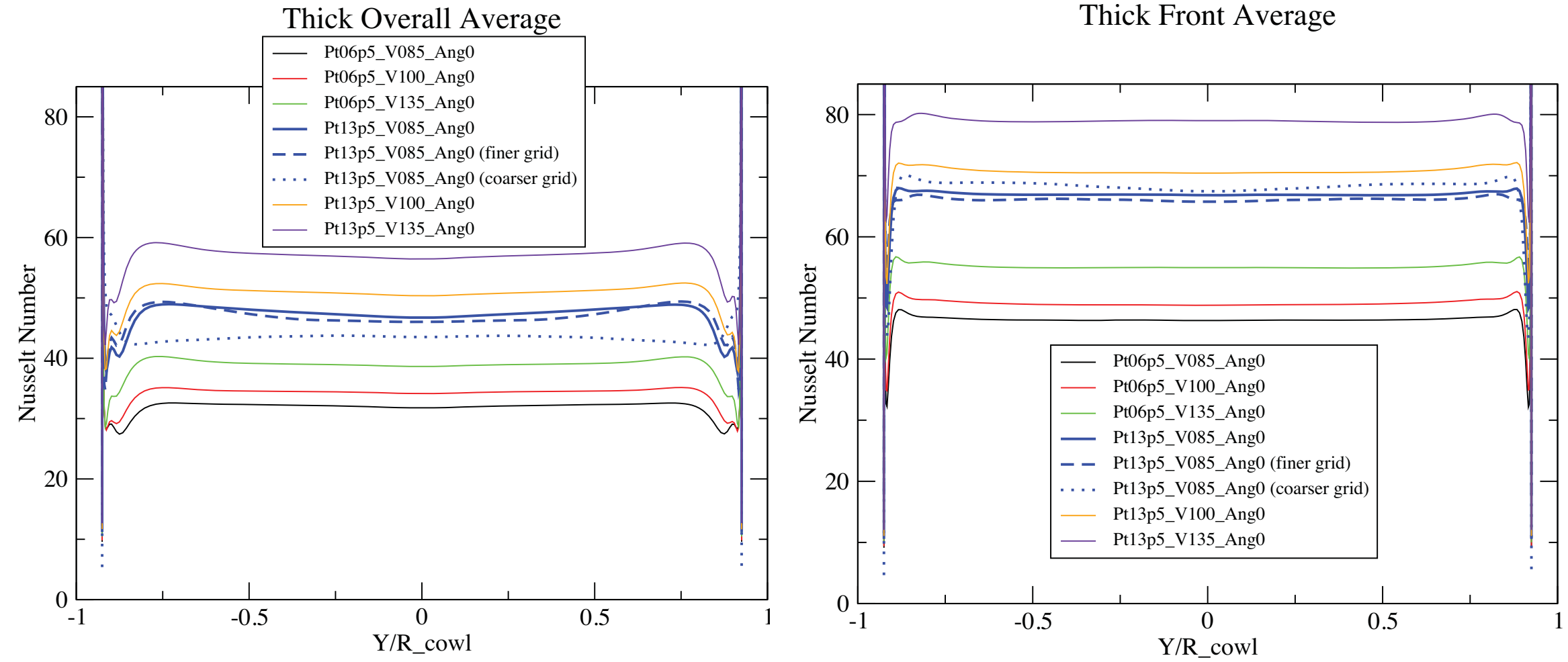


\section{Thin (021) Spanwise Nusselt Number}

Thin Overall Average

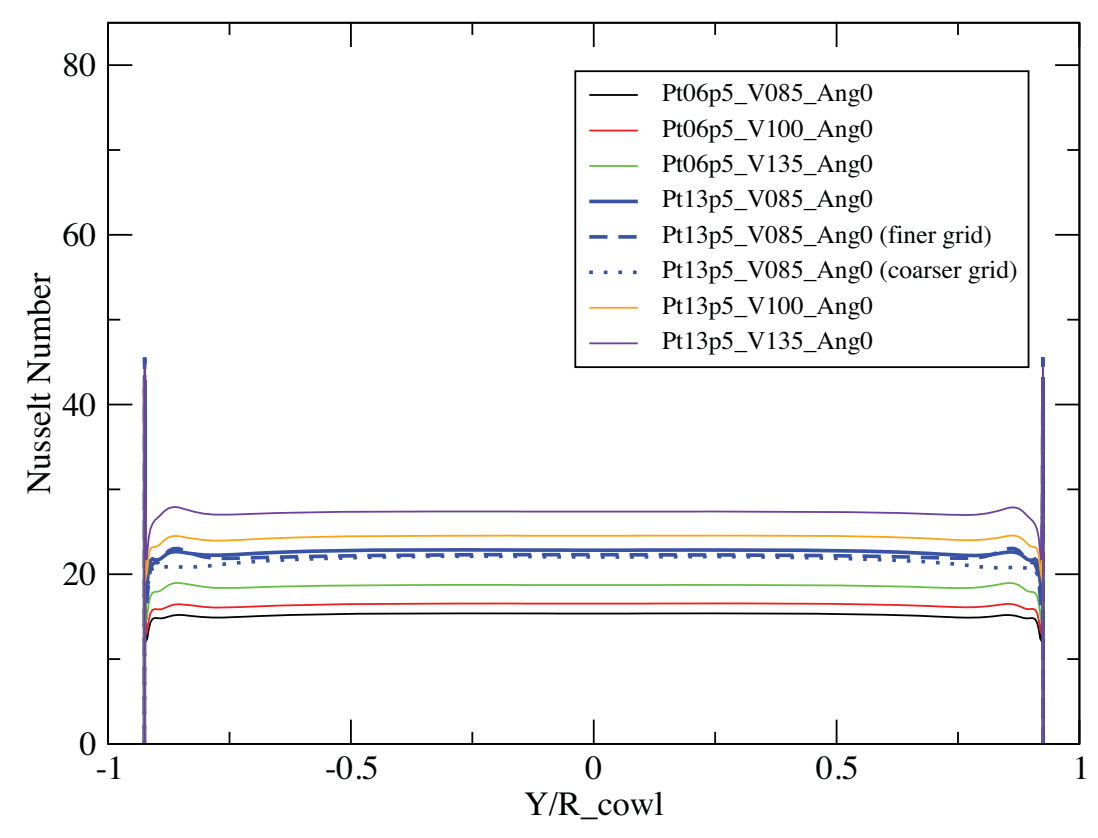

Thin Front Average

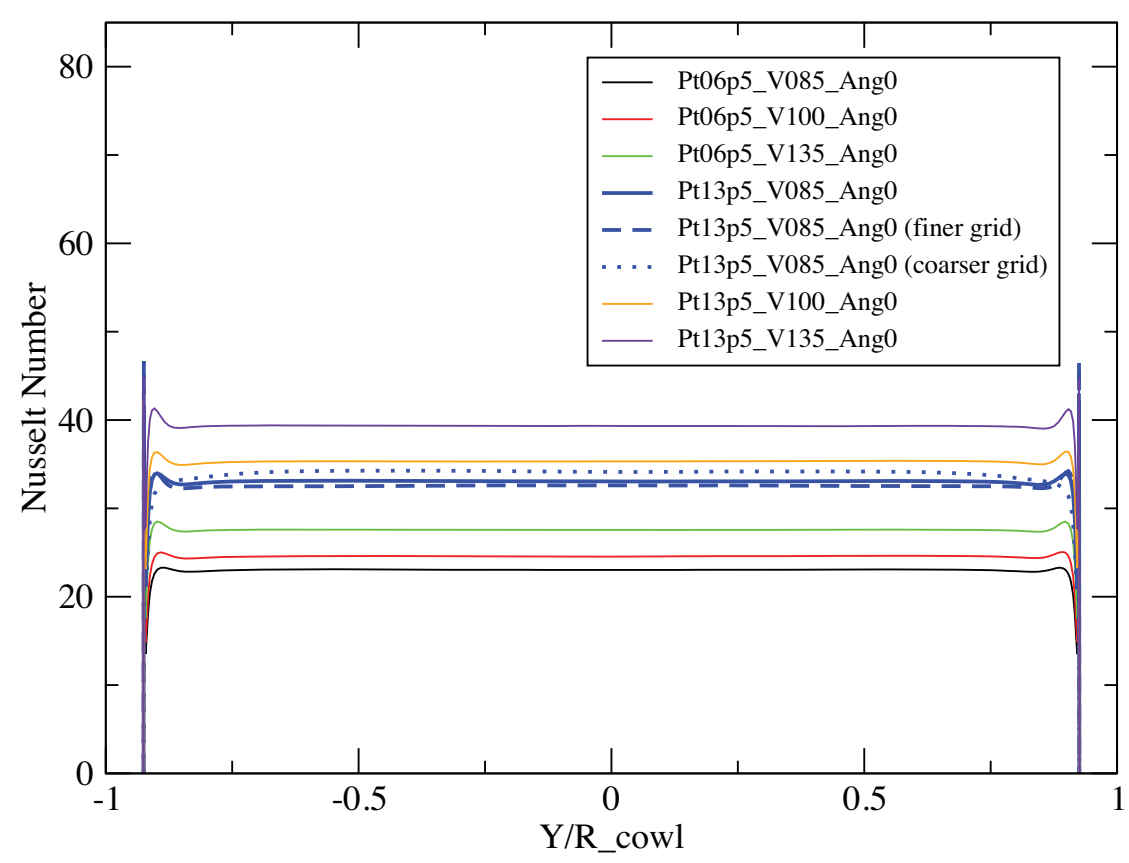




\section{Half-Pipe(HP) Spanwise Nusselt Number}

Scoop Overall Average

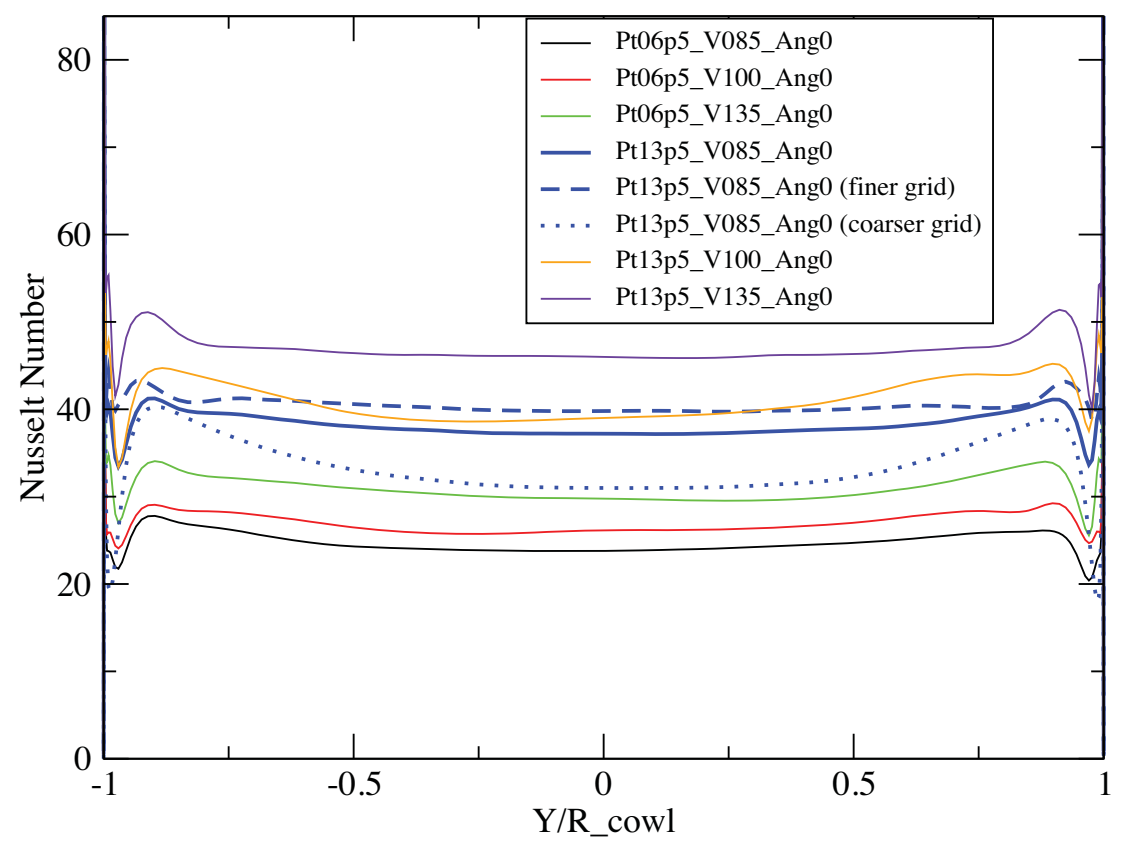

Scoop Front Average

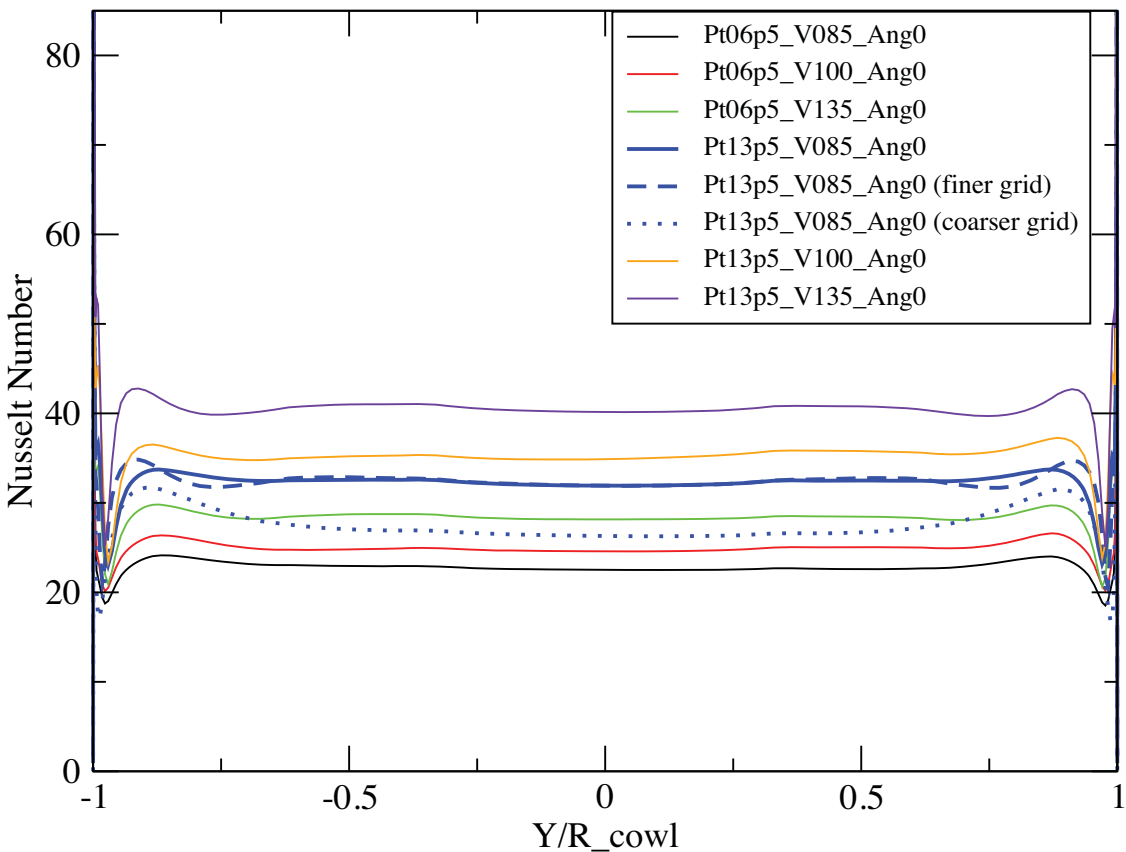


Average Nusselt numbers for Angle of attack, Yaw, and Support cases, compared to straight on at same conditions

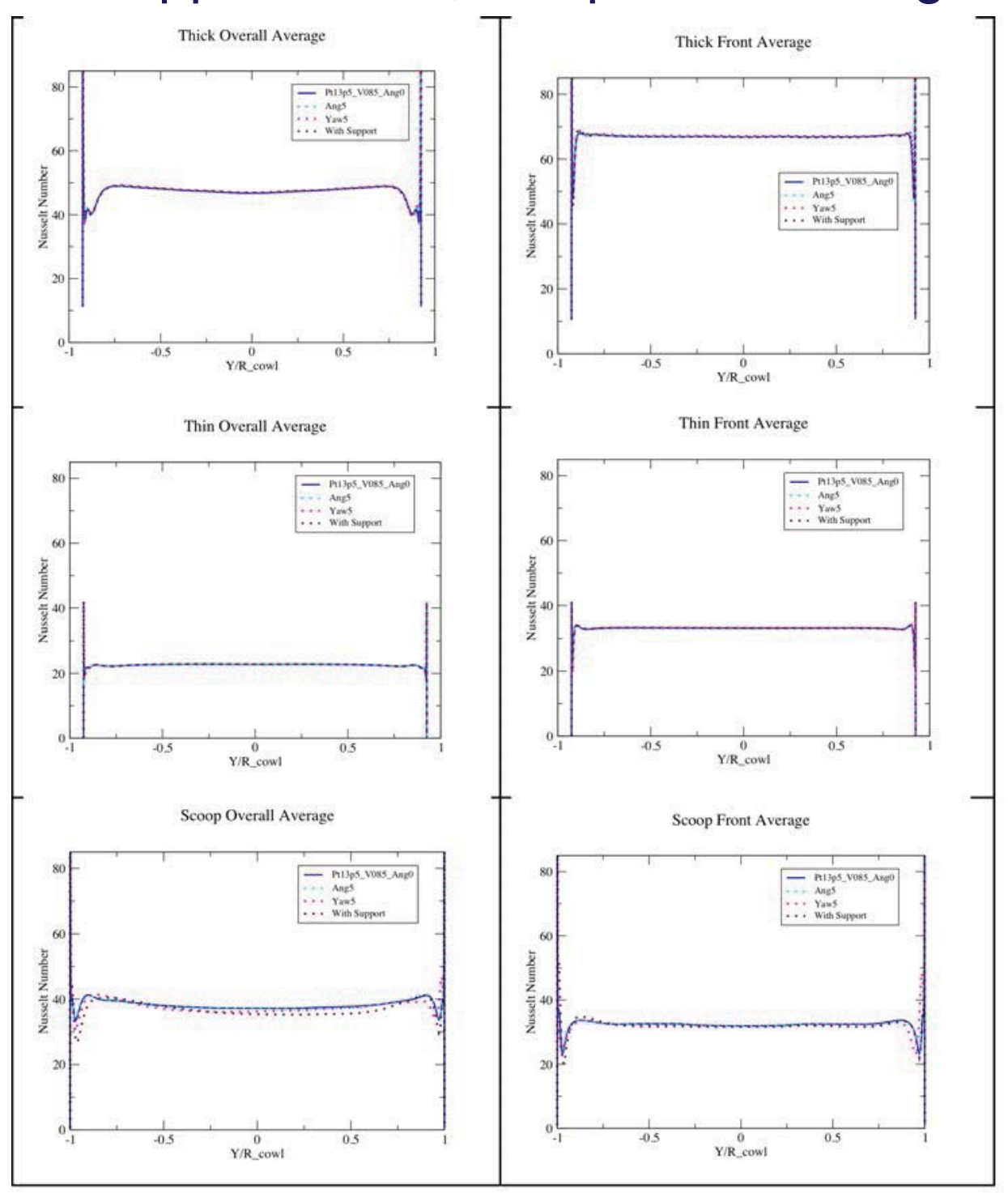

- Very little change due to pitch, yaw, or support strut

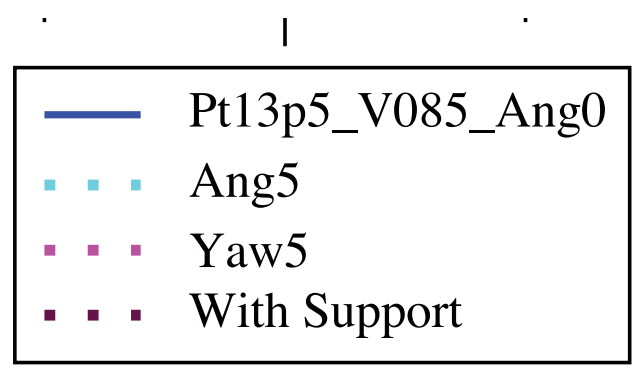


Total collection efficiency results for straight on cases without support Thin (021) Heated Element

\begin{tabular}{|c|c|c|c|r|r|r|r|}
\hline Geometry & Total Pressure & Airspeed & Drop Size & \multicolumn{1}{c|}{ K_0 } & E_m,2D & E_m,3D \\
& psia & $\mathrm{m} / \mathrm{s}$ & Microns & & \multicolumn{1}{c|}{ Lewice2D } & Lewice3D & \\
\hline Thin (021) & 6.5 & 85 & 5 & 8.517 & 0.93108 & 0.82913 & $89.1 \%$ \\
\hline Thin (021) & 6.5 & 85 & 20 & 92.454 & 0.98977 & 0.95719 & $96.7 \%$ \\
\hline Thin (021) & 6.5 & 85 & 50 & 408.181 & 0.99512 & 0.98913 & $99.4 \%$ \\
\hline Thin (021) & 6.5 & 85 & 100 & 1202.878 & 0.99512 & 0.99628 & $100.1 \%$ \\
\hline Thin (021) & 13.5 & 85 & 5 & 7.091 & 0.92325 & 0.81998 & $88.8 \%$ \\
\hline Thin (021) & 13.5 & 85 & 20 & 70.451 & 0.98977 & 0.95366 & $96.4 \%$ \\
\hline Thin (021) & 13.5 & 85 & 50 & 295.481 & 0.99512 & 0.98756 & $99.2 \%$ \\
\hline Thin (021) & 13.5 & 85 & 100 & 842.951 & 0.99512 & 0.99509 & $100.0 \%$ \\
\hline Thin (021) & 13.5 & 135 & 5 & 10.004 & 0.94348 & 0.84129 & $89.2 \%$ \\
\hline Thin (021) & 13.5 & 135 & 20 & 94.972 & 0.98977 & 0.96338 & $97.3 \%$ \\
\hline Thin (021) & 13.5 & 135 & 50 & 388.689 & 0.99512 & 0.99032 & $99.5 \%$ \\
\hline Thin (021) & 13.5 & 135 & 100 & 1092.380 & 0.99512 & 0.99614 & $100.1 \%$ \\
\hline
\end{tabular}


Total collection efficiency results for straight on cases without support Thick (083) Heated Element

\begin{tabular}{|c|c|c|c|c|c|c|c|}
\hline Geometry & Total Pressure & Airspeed & Drop Size & K_o & E_m,2D & E_m,3D & E_m,3D/E_m,2D \\
\hline & psia & $\mathrm{m} / \mathrm{s}$ & Microns & & Lewice2D & Lewice3D & \\
\hline Thick (083) & 6.5 & 85 & 5 & 2.155 & 0.78212 & 0.65481 & $83.7 \%$ \\
\hline Thick (083) & 6.5 & 85 & 20 & 23.388 & 0.97413 & 0.93567 & $96.1 \%$ \\
\hline Thick (083) & 6.5 & 85 & 50 & 103.256 & 0.99226 & 0.98467 & $99.2 \%$ \\
\hline Thick (083) & 6.5 & 85 & 100 & 304.288 & 0.99610 & 0.99472 & $99.9 \%$ \\
\hline Thick (083) & 13.5 & 85 & 5 & 1.794 & 0.75436 & 0.62688 & $83.1 \%$ \\
\hline Thick (083) & 13.5 & 85 & 20 & 17.822 & 0.96767 & 0.92596 & $95.7 \%$ \\
\hline Thick (083) & 13.5 & 85 & 50 & 74.747 & 0.99025 & 0.98096 & $99.1 \%$ \\
\hline Thick (083) & 13.5 & 85 & 100 & 213.238 & 0.99494 & 0.99308 & $99.8 \%$ \\
\hline Thick (083) & 13.5 & 135 & 5 & 2.531 & 0.81610 & 0.68576 & $84.0 \%$ \\
\hline Thick (083) & 13.5 & 135 & 20 & 24.025 & 0.97546 & 0.94182 & $96.6 \%$ \\
\hline Thick (083) & 13.5 & 135 & 50 & 98.325 & 0.99226 & 0.98468 & $99.2 \%$ \\
\hline Thick (083) & 13.5 & 135 & 100 & 276.335 & 0.99543 & 0.99423 & $99.9 \%$ \\
\hline
\end{tabular}


Total collection efficiency results for straight on cases without support Half-Pipe (HP) Heated Element

\begin{tabular}{|c|c|c|c|c|c|c|c|}
\hline Geometry & Total Pressure & Airspeed & Drop Size & K_O & E_m,2D & $E \_m, 3 D$ & E_m,3D/E_m,2D \\
\hline Half-Pipe & 6.5 & 85 & 5 & 2.155 & 0.89161 & 0.72710 & $81.5 \%$ \\
\hline Half-Pipe & 6.5 & 85 & 50 & 103.256 & 0.99208 & 0.98639 & $99.4 \%$ \\
\hline Half-Pipe & 6.5 & 85 & 100 & 304.288 & 0.99409 & 0.99570 & $100.2 \%$ \\
\hline Half-Pipe & 13.5 & 85 & 20 & 17.822 & 0.97909 & 0.93487 & $95.5 \%$ \\
\hline Half-Pipe & 13.5 & 85 & 50 & 74.747 & 0.99141 & 0.98320 & $99.2 \%$ \\
\hline Half-Pipe & 13.5 & 85 & 100 & 213.238 & 0.99409 & 0.99419 & $100.0 \%$ \\
\hline Half-Pipe & 13.5 & 135 & 5 & 2.531 & 0.89161 & 0.75664 & $84.9 \%$ \\
\hline
\end{tabular}




\section{Total collection efficiency results for straight on cases without support}

\begin{tabular}{|c|c|c|c|c|c|c|c|}
\hline Geometry & Total Pressure & Airspeed & Drop Size & K_O & E_m,2D & E_m,3D & E_m,3D/E_m,2D \\
\hline & psia & $\mathrm{m} / \mathrm{s}$ & Microns & & Lewice2D & Lewice3D & \\
\hline Thin (021) & 6.5 & 85 & 5 & 8.517 & 0.93108 & 0.82913 & $89.1 \%$ \\
\hline Thin (021) & 6.5 & 85 & 20 & 92.454 & 0.98977 & 0.95719 & $96.7 \%$ \\
\hline Thin (021) & 6.5 & 85 & 50 & 408.181 & 0.99512 & 0.98913 & $99.4 \%$ \\
\hline Thin (021) & 6.5 & 85 & 100 & 1202.878 & 0.99512 & 0.99628 & $100.1 \%$ \\
\hline Thin (021) & 13.5 & 85 & 5 & 7.091 & 0.92325 & 0.81998 & $88.8 \%$ \\
\hline Thin (021) & 13.5 & 85 & 20 & 70.451 & 0.98977 & 0.95366 & $96.4 \%$ \\
\hline Thin (021) & 13.5 & 85 & 50 & 295.481 & 0.99512 & 0.98756 & $99.2 \%$ \\
\hline Thin (021) & 13.5 & 85 & 100 & 842.951 & 0.99512 & 0.99509 & $100.0 \%$ \\
\hline Thin (021) & 13.5 & 135 & 5 & 10.004 & 0.94348 & 0.84129 & $89.2 \%$ \\
\hline Thin (021) & 13.5 & 135 & 20 & 94.972 & 0.98977 & 0.96338 & $97.3 \%$ \\
\hline Thin (021) & 13.5 & 135 & 50 & 388.689 & 0.99512 & 0.99032 & $99.5 \%$ \\
\hline Thin (021) & 13.5 & 135 & 100 & 1092.380 & 0.99512 & 0.99614 & $100.1 \%$ \\
\hline Thick (083) & 6.5 & 85 & 5 & 2.155 & 0.78212 & 0.65481 & $83.7 \%$ \\
\hline Thick (083) & 6.5 & 85 & 20 & 23.388 & 0.97413 & 0.93567 & $96.1 \%$ \\
\hline Thick (083) & 6.5 & 85 & 50 & 103.256 & 0.99226 & 0.98467 & $99.2 \%$ \\
\hline Thick (083) & 6.5 & 85 & 100 & 304.288 & 0.99610 & 0.99472 & $99.9 \%$ \\
\hline Thick (083) & 13.5 & 85 & 5 & 1.794 & 0.75436 & 0.62688 & $83.1 \%$ \\
\hline Thick (083) & 13.5 & 85 & 20 & 17.822 & 0.96767 & 0.92596 & $95.7 \%$ \\
\hline Thick (083) & 13.5 & 85 & 50 & 74.747 & 0.99025 & 0.98096 & $99.1 \%$ \\
\hline Thick (083) & 13.5 & 85 & 100 & 213.238 & 0.99494 & 0.99308 & $99.8 \%$ \\
\hline Thick (083) & 13.5 & 135 & 5 & 2.531 & 0.81610 & 0.68576 & $84.0 \%$ \\
\hline Thick (083) & 13.5 & 135 & 20 & 24.025 & 0.97546 & 0.94182 & $96.6 \%$ \\
\hline Thick (083) & 13.5 & 135 & 50 & 98.325 & 0.99226 & 0.98468 & $99.2 \%$ \\
\hline Thick (083) & 13.5 & 135 & 100 & 276.335 & 0.99543 & 0.99423 & $99.9 \%$ \\
\hline Half-Pipe & 6.5 & 85 & 5 & 2.155 & 0.89161 & 0.72710 & $81.5 \%$ \\
\hline Half-Pipe & 6.5 & 85 & 20 & 23.388 & 0.98309 & 0.94268 & $95.9 \%$ \\
\hline Half-Pipe & 6.5 & 85 & 50 & 103.256 & 0.99208 & 0.98639 & $99.4 \%$ \\
\hline Half-Pipe & 6.5 & 85 & 100 & 304.288 & 0.99409 & 0.99570 & $100.2 \%$ \\
\hline Half-Pipe & 13.5 & 85 & 5 & 1.794 & 0.85435 & 0.70295 & $82.3 \%$ \\
\hline Half-Pipe & 13.5 & 85 & 20 & 17.822 & 0.97909 & 0.93487 & $95.5 \%$ \\
\hline Half-Pipe & 13.5 & 85 & 50 & 74.747 & 0.99141 & 0.98320 & $99.2 \%$ \\
\hline Half-Pipe & 13.5 & 85 & 100 & 213.238 & 0.99409 & 0.99419 & $100.0 \%$ \\
\hline Half-Pipe & 13.5 & 135 & 5 & 2.531 & 0.89161 & 0.75664 & $84.9 \%$ \\
\hline Half-Pipe & 13.5 & 135 & 20 & 24.025 & 0.98309 & 0.94940 & $96.6 \%$ \\
\hline Half-Pipe & 13.5 & 135 & 50 & 98.325 & 0.99208 & 0.98676 & $99.5 \%$ \\
\hline Half-Pipe & 13.5 & 135 & 100 & 276.335 & 0.99409 & 0.99534 & $100.1 \%$ \\
\hline
\end{tabular}


Local Collection Efficiency for several particle sizes (straight on cases).

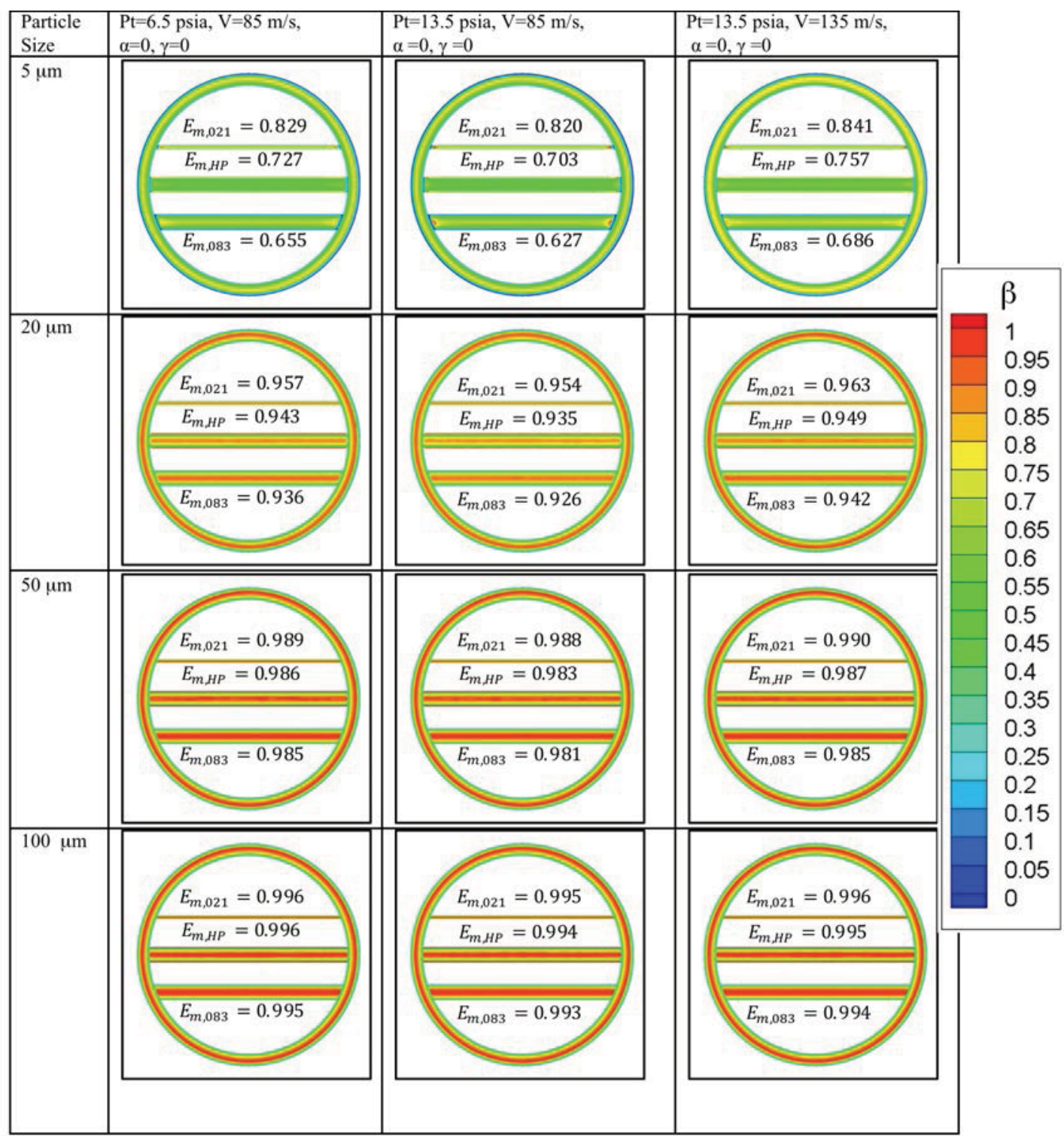




\section{Collection efficiency. Splashing compared to no splashing (straight on cases)}
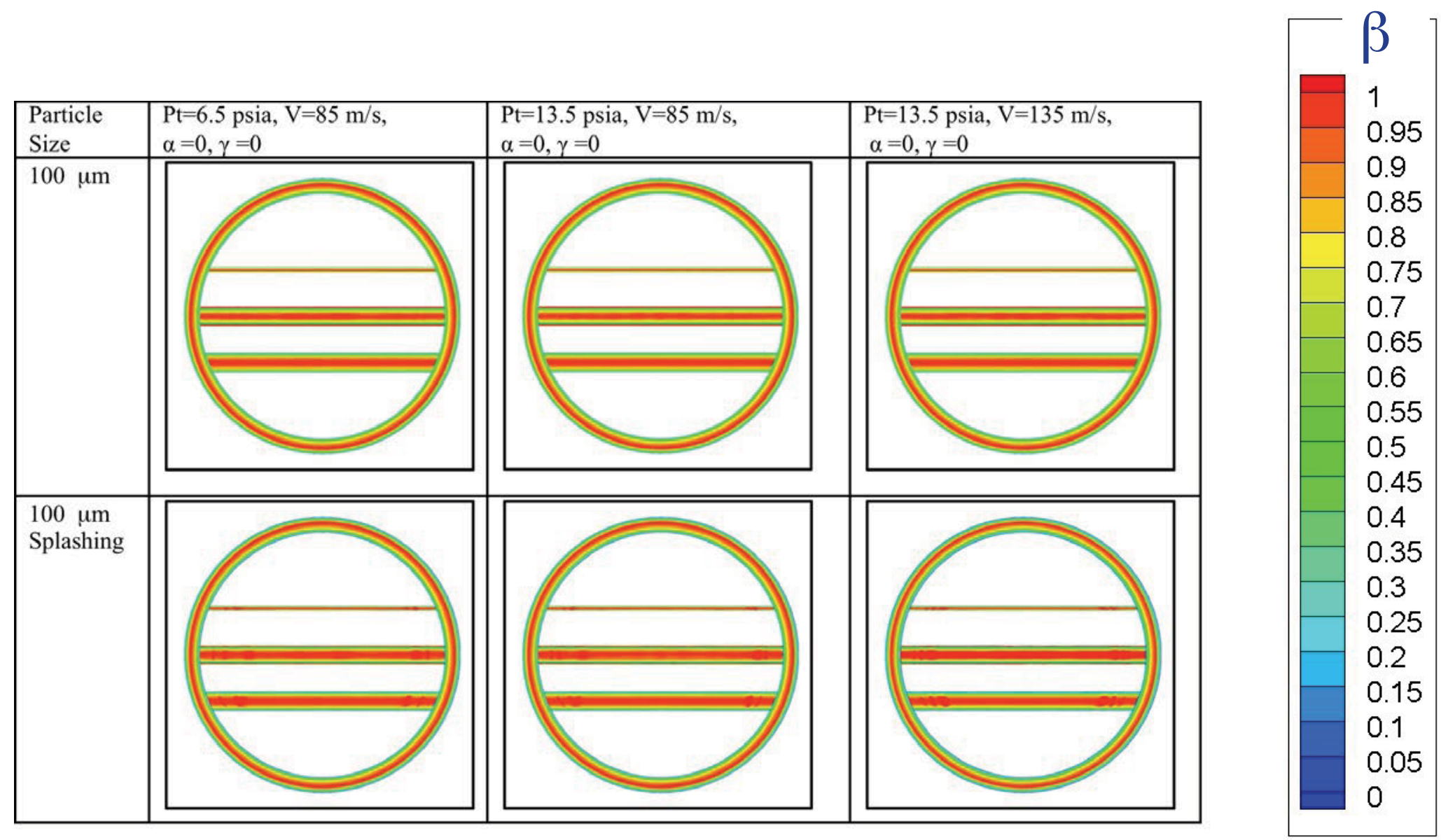


\section{Collection efficiency. Splashing compared to no splashing (angle of attack, yaw, and support cases}
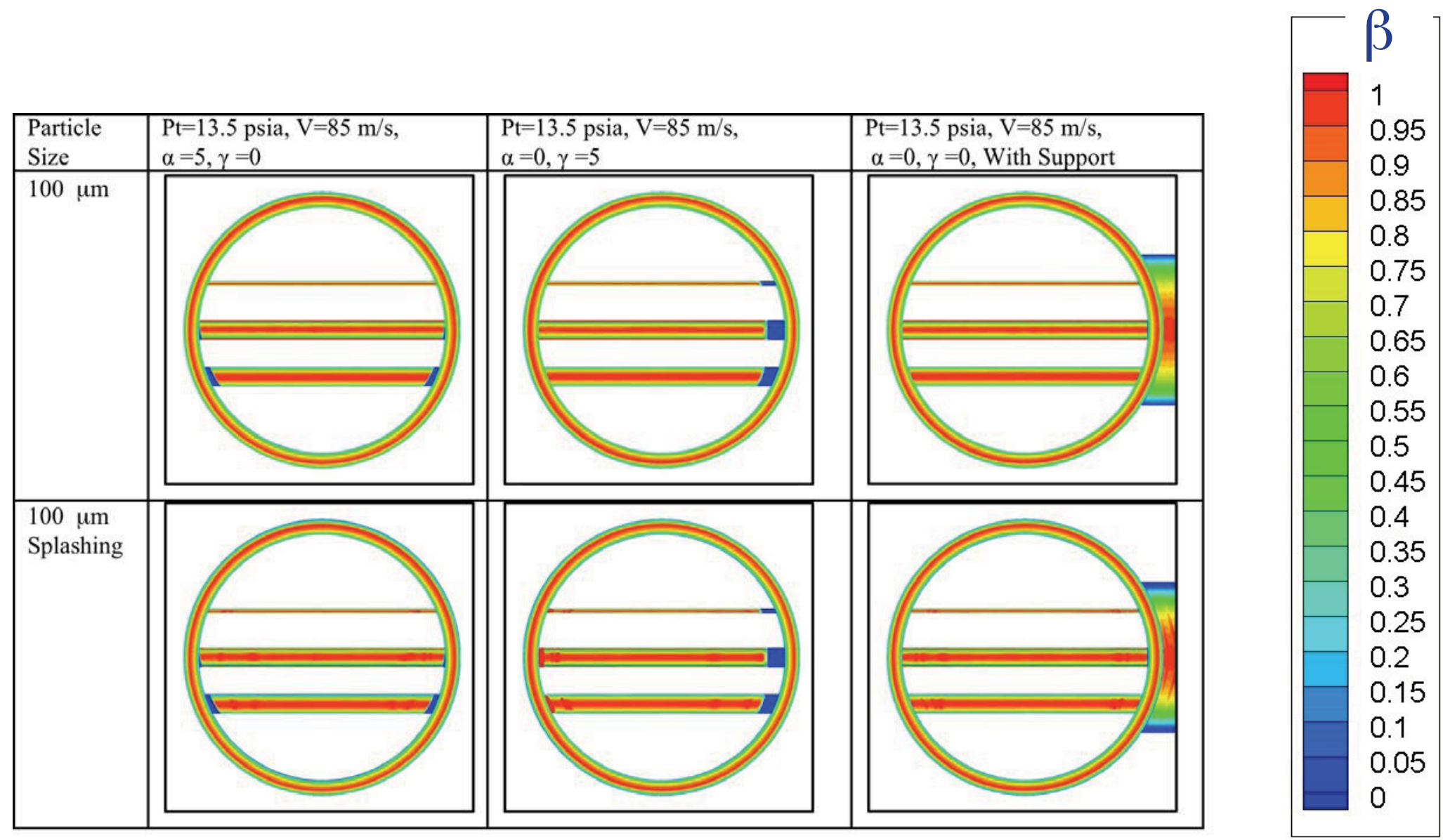